\title{
SPATIAL VARIATIONS OF DUST ABUNDANCES ACROSS THE LARGE MAGELLANIC CLOUD
}

\author{
Déborah Paradis ${ }^{1}$, William T. Reach ${ }^{1}$, Jean-Philippe Bernard ${ }^{2,3}$, Miwa Block ${ }^{4}$, Chad W. Engelbracht ${ }^{4}$, \\ Karl Gordon ${ }^{4}$, Joseph L. Hora ${ }^{5}$, Remy Indebetouw ${ }^{6}$, Akiko Kawamura ${ }^{7}$, Marilyn Meade ${ }^{8}$, Margaret Meixner $^{9}$, \\ Marta Sewilo ${ }^{9}$, Uma P. Vijh ${ }^{10}$, and Kevin VolK ${ }^{11}$ \\ ${ }^{1}$ Infrared Processing and Analysis Center, California Institute of Technology, Pasadena, CA 91125, USA \\ ${ }^{2}$ Université de Toulouse, UPS, CESR, 9 av. du Colonel Roche, F-31028 Toulouse cedex 9, France \\ ${ }^{3}$ CNRS, UMR5187, F-31028 Toulouse, France \\ ${ }^{4}$ Steward Observatory, University of Arizona, 933 North Cherry Avenue, Tucson, AZ 85721, USA \\ ${ }^{5}$ Harvard-Smithsonian Center for Astrophysics, 60 Garden St., MS-65, Cambridge, MA, 02138-1516, USA \\ 67 National Radio Astronomy Observatory and Department of Astronomy, University of Virginia, P.O. Box 3818, Charlottesville, VA 22903-0818, USA \\ ${ }^{7}$ Nagoya University, Department of Astrophysics, Chikusa-Ku, Nagoya, 464-01, Japan \\ ${ }^{8}$ Department of Astronomy, University of Wisconsin, Madison, 475 N. Charter St., Madison, WI 53706, USA \\ ${ }^{9}$ Space Telescope Science Institute, 3700 San Martin Drive, Baltimore, MD 21218, USA \\ ${ }^{10}$ Ritter Astrophysical Research Center, University of Toledo, Toledo, OH 43606, USA \\ ${ }^{11}$ Gemini Observatory, Northern Operations Center, 670 N. Aohuku Place, Hilo, HI 96720, USA \\ Received 2009 February 13; accepted 2009 April 28; published 2009 June 1
}

\begin{abstract}
Using the data obtained with the Spitzer Space Telescope as part of the Surveying the Agents of a Galaxy's Evolution (SAGE) legacy survey, we have studied the variations of the dust composition and abundance across the Large Magellanic Cloud (LMC). Such variations are expected, as the explosive events which have lead to the formation of the many $\mathrm{H}$ i shells observed should have affected the dust properties. Using a model and comparing with a reference spectral energy distribution from our Galaxy, we deduce the relative abundance variations of small dust grains across the LMC. We examined the infrared color ratios as well as the relative abundances of very small grains (VSGs) and polycyclic aromatic hydrocarbons (PAHs) relative to the big grain abundance. Results show that each dust component could have different origins or evolution in the interstellar medium (ISM). The VSG abundance traces the star formation activity and could result from shattering of larger grains, whereas the PAH abundance increases around molecular clouds as well as in the stellar bar, where they could have been injected into the ISM during mass loss from old stars.
\end{abstract}

Key words: dust, extinction - ISM: abundances - infrared: ISM - Magellanic Clouds

\section{INTRODUCTION}

The Large Magellanic Cloud (LMC) is the nearest galaxy external to the Milky Way (MW), located at a distance of about $55 \mathrm{kpc}$, and is oriented with an advantageous almost face-on viewing angle $\left(35^{\circ}\right.$; van der Marel \& Cioni 2001). The LMC is a dwarf galaxy orbiting around the MW, containing more than 30 billion stars. As opposed to the situation in the Galaxy, where studies of the infrared emission from dust in the interstellar medium (ISM) suffers from significant confusion along the line of sight, the favorable viewing angle for the LMC offers a direct view to the processes taking place in the diffuse ISM. The LMC has a lower metallicity than that of our Galaxy, with estimated values close to $0.3-0.5 Z_{\odot}$ (Westerlund 1997) or $0.25 Z_{\odot}$ (Dufour $1984)$; specifically for carbon and oxygen, $Z_{\mathrm{LMC}}^{\mathrm{C}}=0.28 Z_{\odot}^{\mathrm{C}}$ and $Z_{\mathrm{LMC}}^{\mathrm{O}}=0.54 Z_{\odot}^{\mathrm{O}}$.

Extinction curves in several regions of the LMC (Clayton \& Martin 1985; Sauvage \& Vigroux 1991; Gordon et al. 2003) also differ from those measured in the Galaxy. For example, the ultraviolet (UV) bump at $2175 \AA$ is weaker in the LMC than in the MW. Moreover, the far-ultraviolet rise is steeper in the LMC, indicating that grains responsible for this feature could be more abundant. However, the LMC and MW extinction curves are similar in the visible (VIS) and the near-infrared (NIR). The N HI/Av ratio values (Gordon et al. 2003; Cox et al. 2006) indicate a gas/dust ratio ranging between two and four times the Galactic value, depending on the line of sight. Sakon et al. (2006) analyzed the LMC far-infrared (FIR) emission using DIRBE data and showed the existence of some pixels with very bright emission at $240 \mu \mathrm{m}$ which could possibly be interpreted as cold dust. Emission in DIRBE bands at 12 and $25 \mu \mathrm{m}$ normalized to FIR brightness was found to be relatively lower in the LMC than in our Galaxy. These results can be interpreted as a polycyclic aromatic hydrocarbon (PAH) and very small grain (VSG) deficit in the LMC. Sauvage \& Vigroux (1991) studied the Infrared Astronomical Satellite (IRAS) $I_{12} / I_{25}$ versus $I_{60} / I_{100}$ ratio in the LMC and SMC and compared them with observations of several galaxies by Helou (1986). They showed that there is a decrease of the $12 \mu \mathrm{m}$ emission relative to the 60 and $100 \mu \mathrm{m}$ emission associated with the decrease of the metallicity in the LMC and Small Magellanic Cloud (SMC) compared to the MW. They proposed that the low metallicity in the LMC and SMC could limit small grain formation, concluding that the metallicity could act preferentially on the abundance of one grain component.

Bernard et al. (2008) performed a global preliminary analysis of the entire LMC using new Spitzer images (Meixner et al. 2006) covering the entire LMC with much higher angular resolution than the previous IRAS or DIRBE data. They produced a temperature map for the overall LMC and found temperature variations in the range 12.1-34.7 $\mathrm{K}$. They studied three individual regions, determining and modeling their spectral energy distributions (SEDs). They showed that the relative abundance of the dust components is close to that in our Galaxy, in contrast to the previous IRAS and DIRBE studies. They also found that the PAH and VSG abundances are higher in the molecular phase than in the neutral phase for a few selected regions. They explained this result as an increase of the two types of 
grains in unresolved halos surrounding molecular clouds. They also evidenced the presence of an excess of the mid-infrared (MIR) emission in the LMC (70 $\mu$ m excess) which is localized mostly in the atomic medium and in a large circular region around 30-Doradus. They modeled the excess using a modified size distribution for the VSGs and interpreted it as a possible sign of the shattering of larger dust grains into smaller VSGs.

Even if we can interpret infrared color variations as abundance variations, we do not know in general what the origin of these variations is, nor where the different types of grains are formed. Grains could be destroyed in supernova shocks, but grain growth probably takes place in dense molecular clouds through grain coagulation. It is widely accepted (e.g., Allamandola et al. 1989; Matsuura et al. 2008) that PAH molecules could be formed in the expanding envelopes of carbon-rich stars. The objective of our work is to trace dust abundance variations across the LMC in order to gather information about the dust formation and evolution in the ISM.

Sections 2 and 3 briefly summarize the observations and the processing we applied to the Spitzer images. In Section 4, we use an empirical method to study the infrared color ratios. In Section 5 , we focus on the dust abundance variations across the LMC using a dust emission model. Sections 6 and 7 are devoted to discussion and conclusion.

\section{LMC OBSERVATIONS}

\subsection{Spitzer Space Telescope Observations}

The Spitzer Space Telescope (Werner et al. 2004) carries three instruments: the Infrared Array Camera (IRAC; Fazio et al. 2004) which provides imaging in the near and MIR domain (3.6, 4.5, 5.8, and $8.0 \mu \mathrm{m})$, the Infrared Spectrograph (IRS; Houck et al. 2004) which provides both high- and low-resolution spectroscopy at MIR wavelengths, and the Multiband Imaging Photometer for Spitzer (MIPS; Rieke et al. 2004) providing imaging and limited spectroscopic capabilities at three FIR wavelengths $(24,70$, and $160 \mu \mathrm{m})$. IRAC data have an angular resolution of 1".6-1.9 from 3.6 to $8 \mu \mathrm{m}$, and MIPS data have an angular resolution of $6^{\prime \prime}, 18^{\prime \prime}$, and $40^{\prime \prime}$ at 24,70 , and $160 \mu \mathrm{m}$, respectively.

The data were obtained as part of the Surveying the Agents of a Galaxy's Evolution (SAGE) Spitzer legacy survey (Meixner et al. 2006). The survey covered a $\sim 7 \times 7 \mathrm{deg}$ region centered on the LMC with all of the IRAC and MIPS bands. We used mosaics which combined both epochs of the data into a final map at a resolution of $3^{\prime \prime} .6$ pixel $^{-1}$ for IRAC, and 2.49, 4.8, and $15^{\prime \prime} .6$ pixel $^{-1}$ for the MIPS 24, 70, and $160 \mu \mathrm{m}$ maps, respectively.

\subsection{IRIS Data}

The IRIS data are the improved version of the latest IRAS images, corresponding to the second generation processing (or IRAS Sky Survey Atlas, ISSA), see Miville-Deschênes \& Lagache (2005). These maps have been reprocessed to improve sensitivity and absolute calibration. Compared to the latest version of the IRAS data, this new product named IRIS (for Improved Reprocessing of the IRAS Survey) benefits from a better zodiacal light subtraction, calibration, and zero level adjustments to match the DIRBE data on large angular scales. With a resolution near $4^{\prime}$, the IRIS survey covers $98 \%$ of the sky at four wavelengths $(12,25,60$, and $100 \mu \mathrm{m})$.

\section{IMAGE PROCESSING}

We briefly describe here the processing we applied to each map. Details of the data treatment used here are given in Bernard et al. (2008). All data were projected on a common grid centered at $\alpha_{2000}=05^{\mathrm{h}} 18^{\mathrm{m}} 48^{\mathrm{s}}$ and $\delta_{2000}=-68^{\circ} 42^{\prime} 00^{\prime \prime}$, with a pixel size of $2^{\prime}$ and then smoothed to the IRIS resolution of $4^{\prime}$. We subtracted the Galactic foreground emission in order to avoid any confusion between the emission from the LMC and from our Galaxy. We used the SED of the high latitude galactic emission of Dwek et al. (1997) (see Bernard et al. 2008), and the H I $21 \mathrm{~cm}$ emission in the heliocentric velocity range $-64<v_{\text {hel }}<100 \mathrm{~km} \mathrm{~s}^{-1}$ (Staveley-Smith et al. 2003). In order to eliminate residual zodiacal emission and cosmic infrared background (CIB), we have subtracted a background from all images corresponding to the median intensity in the region located $4^{\circ}$ from the center of the maps. Point sources in archival catalogs (the Two Micron All Sky Survey (2MASS) catalog for IRAC and the SAGE catalog for MIPS) were masked from the IRAC and MIPS maps before degrading their resolution to $4^{\prime}$, to match that of the IRIS maps. For the IRIS point-source subtraction, we used the SAGE catalogs; specifically the 160, 70,24 , and $8 \mu \mathrm{m}$ catalogs for the IRIS 100, 60, 25, and $12 \mu \mathrm{m}$ maps, respectively. The Spitzer and IRIS images at the $4^{\prime}$ angular resolution are presented in Figures 1 and 2.

\section{INFRARED COLORS}

\subsection{Color-Color Diagrams}

We first examine the trends in the LMC color-color diagrams (see Figure 3 ). The $I_{8} / I_{160}$ and $I_{24} / I_{160}$ ratios can be considered as proxies for the PAH and VSG relative abundances with respect to that of the big grains (BGs), respectively. The $I_{100 /}$ $I_{160}$ ratio is directly linked to $\mathrm{BG}$ equilibrium temperature and therefore to the interstellar radiation field (ISRF) intensity. The $I_{8} / I_{160}$ and $I_{24} / I_{160}$ ratios show a trend with the $I_{100} / I_{160}$ ratio, such that the ratios increase with the BG temperature. However, in the first case, the dispersion is quite large, whereas in the second case the trend is more pronounced. We can conclude that the PAH abundance does not depend strongly on the radiation field intensity. The VSG abundance, however, seems to be increased in warm regions of the LMC.

To connect the observed brightnesses to dust abundances more precisely, we use an updated version of the Désert et al. (1990) model, which includes an improved description of the PAH IR emission bands at 3.3, 6.2, 7.7, 8.6, 11.3, 12.7, and $16.4 \mu \mathrm{m}$. This version also takes into account the results of Flagey et al. (2006), based on the work by Rapacioli et al. (2005) including the cross sections of neutral and ionized PAHs. We assume the same ISRF spectral shape as in the solar neighborhood. We show in Figure 4 the variations of the predicted $I_{24} / I_{160}$ and $I_{8} / I_{160}$ with $I_{70} / I_{160}$ for different values of ISRF intensity ( $X_{\mathrm{ISRF}}$ ), from 0.2 to about 42 , and various VSG and PAH mass abundances relative to hydrogen. The reference MW dust abundances from Désert et al. (1990) are $Y_{\mathrm{BG}}=6.4 \times 10^{-3}, Y_{\mathrm{VSG}}=4.7 \times 10^{-4}, Y_{\mathrm{PAH}}=4.3 \times 10^{-4}$, where $Y_{X}$ is the mass of dust species $X$ relative to $H$. In Figure 4, we allowed the $Y_{\mathrm{VSG}}$ and $Y_{\mathrm{PAH}}$ parameters to vary from $5 \times 10^{-6}$ to 0.5 . Comparing the values of the $I_{8} / I_{160}$, $I_{24} / I_{160}$, and $I_{70} / I_{160}$ ratios given in Table 1 to the predicted values deduced from the model, we deduce from Figure 4 that the intensity of the ISRF in the diffuse LMC medium is about $4.5<X_{\mathrm{ISRF}}<6.5$, taking into account the errors on the brightness ratios. Similarly, the values of the VSG and PAH mass 

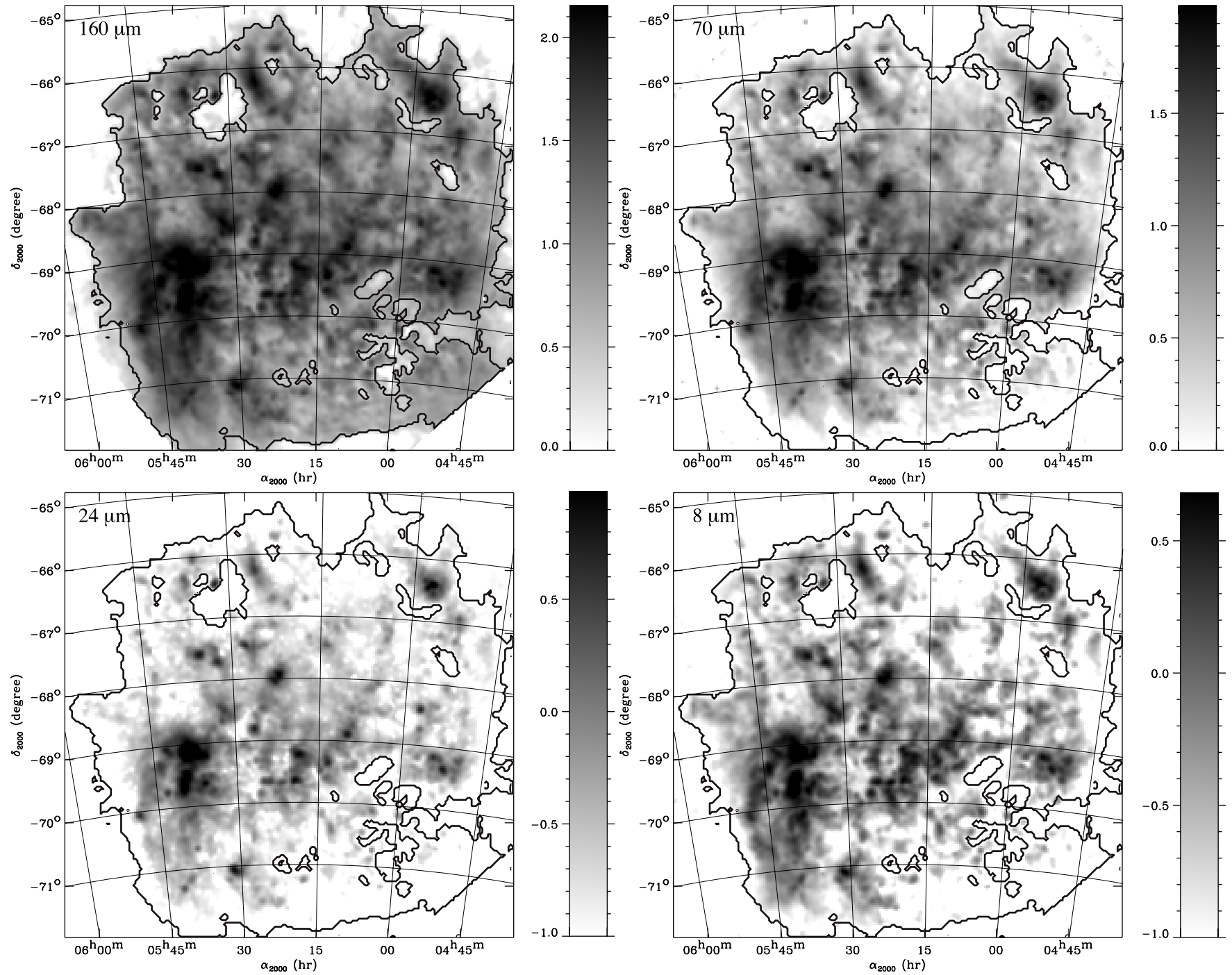

Figure 1. Spitzer images at $160,70,24$, and $8 \mu \mathrm{m}$, at the $4^{\prime}$ angular resolution, after the processing described in Section 3 . The gray scale is $\log _{10}\left(I_{\nu}\left(\mathrm{MJy} \mathrm{sr}^{-1}\right)\right)$.

abundance are in the range $3.5 \times 10^{-4}<Y_{\mathrm{VSG}}<4.7 \times 10^{-4}$ and $3.5 \times 10^{-4}<Y_{\mathrm{PAH}}<4.3 \times 10^{-4}$, respectively. The diffuse ISM in the LMC is therefore subjected to an ISRF which is moderately stronger than that in the solar neighborhood, and appears to contain a similar amount of VSGs and PAHs with respect to large grains, once the effect of heating has been properly taken into account.

\subsection{Infrared Correlations}

In order to measure the color ratios, we performed linear correlations between the IR brightnesses over the whole LMC, using

$$
\begin{aligned}
& I\left(\lambda_{1}\right)=a_{1} I\left(\lambda_{2}\right)+b_{1}, \\
& I\left(\lambda_{2}\right)=a_{2} I\left(\lambda_{1}\right)+b_{2},
\end{aligned}
$$

where $a_{1}$ and $a_{2}$ are the color ratio parameters to be determined and $b_{1}$ and $b_{2}$ are constants corresponding to eventual residual offsets in the images. To avoid any bias in the results depending on the order of the axis we consider, we take the mean value between $a_{1}$ and $1 / a_{2}$ and we define the uncertainty on each ratio as the difference between these two values. In order to identify outliers in the fit, we performed iterations of the linear regression and removed pixels distant by more than $3 \sigma$ from the linear fit, where $\sigma$ is the standard deviation of the distance between the data and the fit. This process reached convergence in about 15 iterations. The results of the regression are given in Table 1. A graphical representation of the table is given in Figure 5.

We compare the ratios obtained in the LMC with other ratios calculated in the SMC, in the Galaxy, and in other galaxies. The ratios from Bernard et al. (2008) have been deduced from the total SED in the overall LMC (see their Tables 5 and 7). Therefore in this case, individual $\mathrm{H}$ II regions such as the 30Doradus region are included, which contribute a large fraction of the total brightness. For that reason, our results presented here are different from those obtained by Bernard et al. (2008). The correlation method proposed here is more appropriate to study the diffuse ISM, in the sense that pixels dominated by the diffuse medium are given more importance than the ones dominated by individual $\mathrm{H}$ II regions, which are disregarded as outliers. The quoted color ratios in the SMC are taken from Bot et al. (2004) and have been deduced from Gaussian fits of the histograms of the ratios. We find the $I_{100} / I_{160}$ and $I_{60} / I_{160}$ ratios to be $66 \%$ and $62 \%$ lower, respectively, in the LMC, compared to the $I_{100} / I_{170}$ and $I_{60} / I_{170}$ ratios in the SMC. These differences 

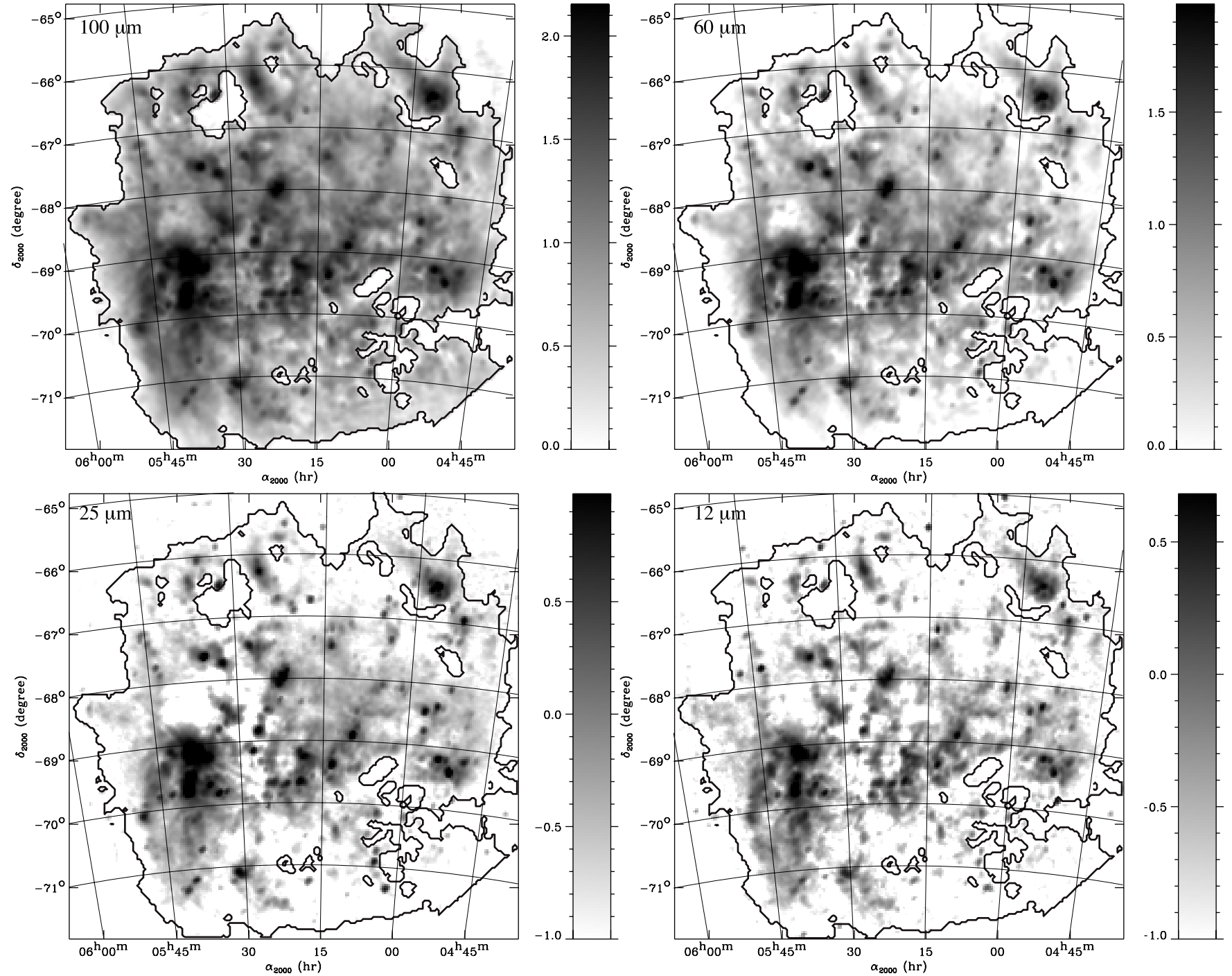

Figure 2. IRIS images at 100, 60, 25, and $12 \mu \mathrm{m}$, at the $4^{\prime}$ angular resolution, after the processing described in Section 3 . The grayscale is $\log _{10}\left(I_{v}\left(\mathrm{MJy} \mathrm{sr}^{-1}\right)\right)$.
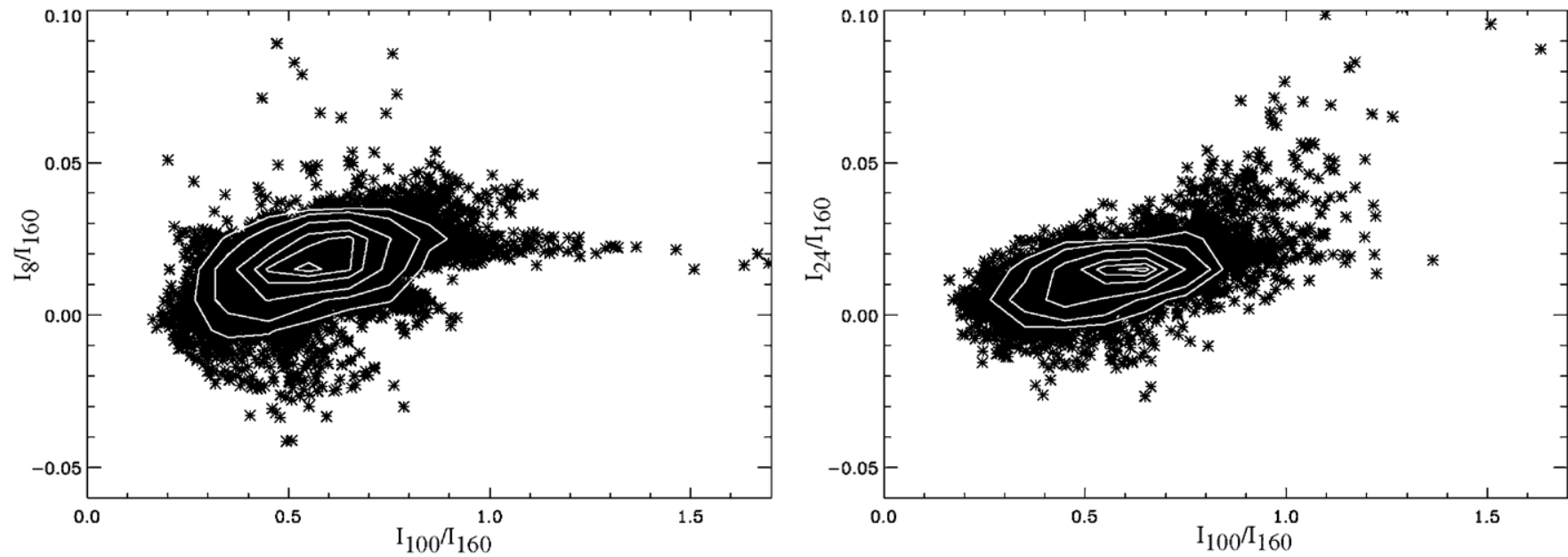

Figure 3. $I_{8} / I_{160} \mu \mathrm{m}$ (left) and $I_{24} / I_{160} \mu \mathrm{m}$ (right) vs. $I_{100} / I_{160} \mu \mathrm{m}$ ratios. Every group of three points has been averaged to produce one plotted point.

must be due to the average equilibrium temperature of the large grains in the SMC being higher than in the LMC. However, the $I_{25} / I_{160}$ and $I_{12} / I_{160}$ ratios, which trace mostly the VSG and PAH abundance relative to BGs and which to first order do not depend on the BG temperature, are almost the same in the two galaxies. Bolatto et al. (2007) explored the southwest region of the bar in the SMC and found an overall $I_{8} / I_{24}$ ratio close to 0.36 , lower by a factor of 4 compared to our results in the LMC. Engelbracht et al. (2005) analyzed this ratio as a function of the metallicity for a sample of 34 galaxies. They 

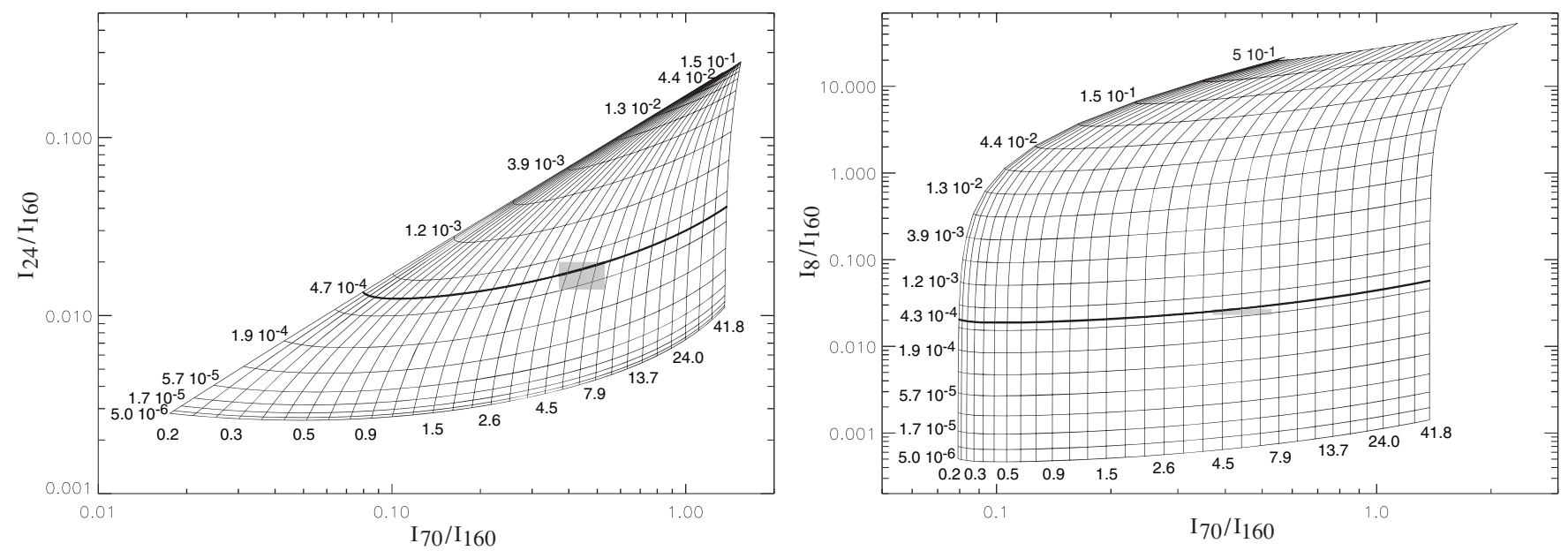

Figure 4. Predicted $I_{24} / I_{160}$ (left) and $I_{8} / I_{160}$ (right) vs. $I_{70} / I_{160}$ using the Désert et al. (1990) model, for various intensities of the ISRF (from 0.2 to 42 ) and VSG mass abundances (left) and PAH mass abundances (right) (from $Y_{\mathrm{VSG}, \mathrm{PAH}}=5 \times 10^{-6}$ to $Y_{\mathrm{VSG}, \mathrm{PAH}}=0.5$ ). The bold lines correspond to the reference MW dust abundances from Désert et al. (1990): $Y_{\mathrm{VSG}}=4.7 \times 10^{-4}$ and $Y_{\mathrm{PAH}}=4.3 \times 10^{-4}$. The gray rectangular boxes show the color values derived for the LMC (see the text). The size of the boxes shows the error.

Table 1

Infrared Color Ratios of Data

\begin{tabular}{|c|c|c|c|c|c|}
\hline IR Ratios & LMC & $\mathrm{LMC}^{\mathrm{a}}$ & $\mathrm{SMC}^{\mathrm{b}}$ & $\begin{array}{c}\text { GC }^{\mathrm{c}} \\
\text { GFLS/ GLIMPSE }^{\mathrm{e}}\end{array}$ & 34 Galaxies $^{\mathrm{d}}$ \\
\hline$I_{100} / I_{160^{\mathrm{f}}}$ & $(6.6 \pm 0.5) 10^{-1}$ & $(7.0 \pm 1.9) 10^{-1}$ & $1.0 \pm 0.2$ & $\ldots$ & $\ldots$ \\
\hline$I_{70} / I_{160}$ & $(4.5 \pm 0.8) 10^{-1}$ & $(4.5 \pm 0.9) 10^{-1}$ & & $\ldots$ & $\ldots$ \\
\hline$I_{60} / I_{160^{\mathrm{f}}}$ & $(3.1 \pm 0.7) 10^{-1}$ & $(3.4 \pm 0.7) 10^{-1}$ & $0.5 \pm 0.1$ & $\ldots$ & $\ldots$ \\
\hline$I_{25} / I_{160^{\mathrm{f}}}$ & $(2.4 \pm 1.0) 10^{-2}$ & $(1.9 \pm 0.6) 10^{-2}$ & $(2.0 \pm 1.0) 10^{-2}$ & $\ldots$ & $\ldots$ \\
\hline$I_{24} / I_{160}$ & $(1.7 \pm 0.3) 10^{-2}$ & $(2.5 \pm 0.5) 10^{-2}$ & $\ldots$ & $\cdots$ & $\cdots$ \\
\hline$I_{12} / I_{160^{\mathrm{f}}}$ & $(1.5 \pm 0.4) 10^{-2}$ & $(7.4 \pm 3.8) 10^{-3}$ & $(1.1 \pm 0.6) 10^{-2}$ & $\ldots$ & $\ldots$ \\
\hline$I_{8} / I_{160}$ & $(2.5 \pm 0.2) 10^{-2}$ & $(1.6 \pm 0.4) 10^{-2}$ & $\ldots$ & $\ldots$ & $\ldots$ \\
\hline$I_{5.8} / I_{160}$ & $(9.2 \pm 7.1) 10^{-3}$ & $(2.7 \pm 0.9) 10^{-3}$ & $\ldots$ & $\ldots$ & $\ldots$ \\
\hline$I_{8} / I_{24}$ & $1.6 \pm 0.3$ & $(6.3 \pm 1.4) 10^{-1}$ & $0.36^{\mathrm{g}}$ & $\ldots$ & $(8.0 \pm 4.0) 10^{-2 \mathrm{~h}}$ \\
\hline$I_{8} / I_{12}$ & $1.7 \pm 0.2$ & $2.1 \pm 1.1$ & $\ldots$ & $\ldots$ & $\ldots$ \\
\hline$I_{5.8} / I_{8}$ & $(3.3 \pm 2.1) 10^{-1}$ & $(1.7 \pm 0.6) 10^{-1}$ & $\ldots$ & $\begin{array}{l}(3.7 \pm 0.3) 10^{-1 \mathrm{c}} \\
(2.6-3.7) 10^{-1 \mathrm{e}}\end{array}$ & $\ldots$ \\
\hline$I_{4.5} / I_{5.8}$ & $(5.4 \pm 7.7) 10^{-1}$ & $(4.3 \pm 28.5) 10^{-2}$ & $\ldots$ & $\ldots$ & $\ldots$ \\
\hline$I_{3.6} / I_{4.5}$ & $(1.6 \pm 0.2) 10^{-1}$ & $1.1 \pm 7.6$ & $\ldots$ & $\ldots$ & \\
\hline$I_{4.5} / I_{8}$ & $(1.8 \pm 3.7) 10^{-1}$ & $(7.4 \pm 5.6) 10^{-3}$ & $\ldots$ & $(3.7-6.5) 10^{-2 \mathrm{e}}$ & $>0.1$ if $\mathrm{I}_{8} / \mathrm{I}_{24}<0.1^{\mathrm{h}}$ \\
\hline$I_{3.6} / I_{8}$ & $(2.9 \pm 6.3) 10^{-3}$ & $(7.9 \pm 7.6) 10^{-3}$ & $\ldots$ & $(5.9-9.4) 10^{-3 e}$ & $\ldots$ \\
\hline
\end{tabular}

Notes.

${ }^{a}$ Bernard et al. (2008) (overall LMC).

b Bot et al. (2004) (overall SMC).

${ }^{\mathrm{c}}$ Galactic center from Arendt et al. (2008).

${ }^{\mathrm{d}}$ Engelbracht et al. (2005).

${ }^{e}$ Galactic First Look Survey/Galactic Legacy Infrared Mid-Plane Survey Extraordinaire from Flagey et al. (2006).

f $170 \mu \mathrm{m}$ instead of $160 \mu \mathrm{m}$ for the Bot et al. (2004) ratios.

${ }^{g}$ Bolatto et al. (2007) (southwest bar region of the SMC).

${ }^{\mathrm{h}}$ In subsolar metallicity environments (with a solar metallicity reference $12+\log (\mathrm{O} / \mathrm{H})=8.7$, Allende Prieto et al. 2001).

show that the $I_{8} / I_{24}$ ratio decreases with metallicity. Dwek \& Galliano (2009) observed the same trend analyzing the emission of several nearby galaxies. They interpreted this as resulting from a lower PAH abundance in low-metallicity environments. That could explain why this ratio is smaller in the SMC, whose metallicity is smaller than that of the LMC. According to Engelbracht et al. (2005), the mean ratio below one-third solar metallicity is $0.08 \pm 0.04$, using a solar metallicity reference of $12+\log (\mathrm{O} / \mathrm{H})=8.7$ (Allende Prieto et al. 2001). For galaxies with metallicity comparable to that of the LMC (between $1 / 3$ and $1 / 2$ solar), we expect from Engelbracht et al. (2005) an $I_{8} / I_{24}$ ratio close to 0.4 . This is significantly lower than the median value of 1.6 we derived here for the diffuse emission in the LMC. The PAH relative abundance therefore seems to be larger in the diffuse ISM of the LMC than in other galaxies with similar low metallicity. However, Bernard et al. (2008) found a ratio similar to Engelbracht et al. (2005), which could indicate that the ratio in external galaxies is affected by the contribution from a major $\mathrm{H}$ II region similar to 30-Doradus in the LMC.

The $I_{5.8} / I_{8}$ ratio has been studied by Arendt et al. (2008) in the Galactic center and by Flagey et al. (2006) in the Galactic diffuse ISM, as observed within the Galactic First Look Survey (GFLS) and Galactic Legacy Infrared Mid-Plane Survey Extraordinaire (GLIMPSE; Churchwell \& the GLIMPSE Team 2005). In the case of the Galactic center, Arendt et al. (2008) found a uniform ratio for the diffuse emission, independent of the $8 \mu \mathrm{m}$ bright- 


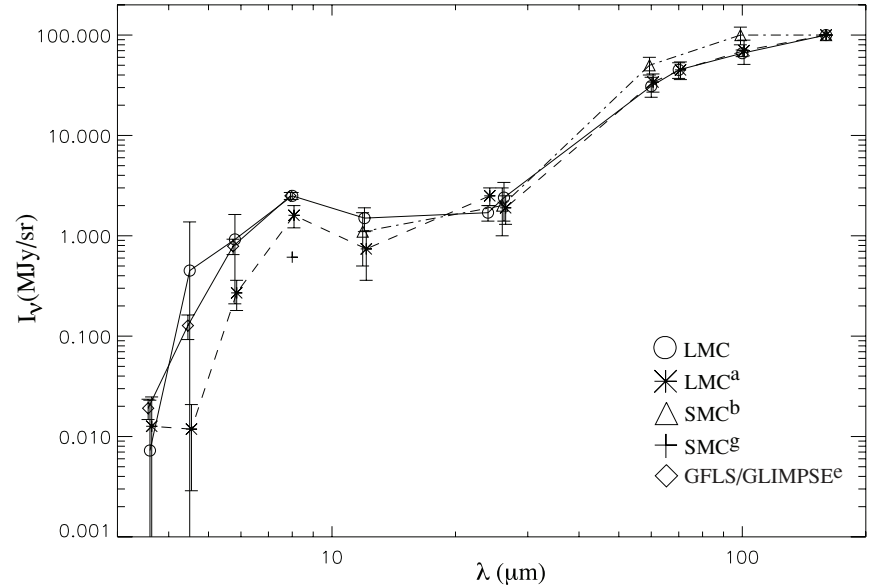

Figure 5. SEDs deduced from the IR ratios given in Table 1, for the LMC. Our results are represented by circles. The SED of the whole LMC by Bernard et al. (2008) is shown by stars. The SMC points are from Bot et al. (2004; triangles) and Bolatto et al. (2007; cross). The Galactic diffuse ISM points are from Flagey et al. (2006; diamonds). A slight shift in wavelengths has been applied for clarity.

ness. Their median value of $I_{5.8} / I_{8}=0.37 \pm 0.03$ is close to our ratio of $0.33 \pm 0.2$. The IRAC ratio obtained by Flagey et al. (2006) ranges from 0.26 to 0.37 and is also in agreement with our result. Therefore, the $I_{5.8} / I_{8}$ ratio seems to be rather similar, around 0.3 , in the LMC and the MW, independent of metallicity.

We find an $I_{4.5} / I_{8}$ ratio in the LMC equal to $0.18 \pm 0.37$. Flagey et al. (2006) found values between $3.7 \times 10^{-2}$ and $6.5 \times 10^{-2}$ in the MW, lower than our result by a factor of 2.8 and 4.9 , respectively. The model we use shows that this ratio is rather independent of the ionization state of the PAHs, increasing by only about $10 \%$ when going from fully neutral to fully ionized PAHs. It is therefore likely that ionization is not the reason for the observed difference. However, the size distribution of PAHs is likely to affect this ratio, leading to larger ratio values for smaller PAH sizes. Therefore, the observed difference could indicate the presence of smaller PAHs in the LMC than in the MW.

For the $I_{3.6} / I_{8}$ ratio, we find in the LMC a value of $(2.9 \pm$ $6.3) \times 10^{-3}$, two to three times lower than the ratio derived by Flagey et al. (2006) (after point-source subtraction). This ratio is sensitive to PAH ionization and decreases by $90 \%$ when going from fully neutral to fully ionized PAHs. Therefore, the observed difference with the MW value could indicate the presence of ionized PAHs in the LMC. Engelbracht et al. (2005) found that in subsolar metallicity galaxies, galaxies with high values of $I_{4.5} / I_{8}(>0.1)$ tend to have low values of $I_{8} / I_{24}$ (essentially below 0.1). In the LMC, we have both large $I_{8} / I_{24}$ and large $I_{4.5} / I_{8}$ ratios and a low metallicity compared to the solar value. The differences could be due to distinct properties of the nearinfrared continuum or of the PAHs. Nevertheless, the emission at $3.6 \mu \mathrm{m}$ is dominated by stellar emission, and therefore we caution that point-source subtraction for these data is difficult and could have an impact on the results.

\section{DUST ABUNDANCE VARIATIONS}

\subsection{Method}

We consider the IR brightness as the sum of the contributions from the three dust components, BG, VSG, and PAH

$$
I_{v}(\lambda)=Y^{\mathrm{BG}} I_{v}^{\mathrm{BG}}(\lambda)+Y^{\mathrm{VSG}} I_{v}^{\mathrm{VSG}}(\lambda)+Y^{\mathrm{PAH}} I_{v}^{\mathrm{PAH}}(\lambda)
$$

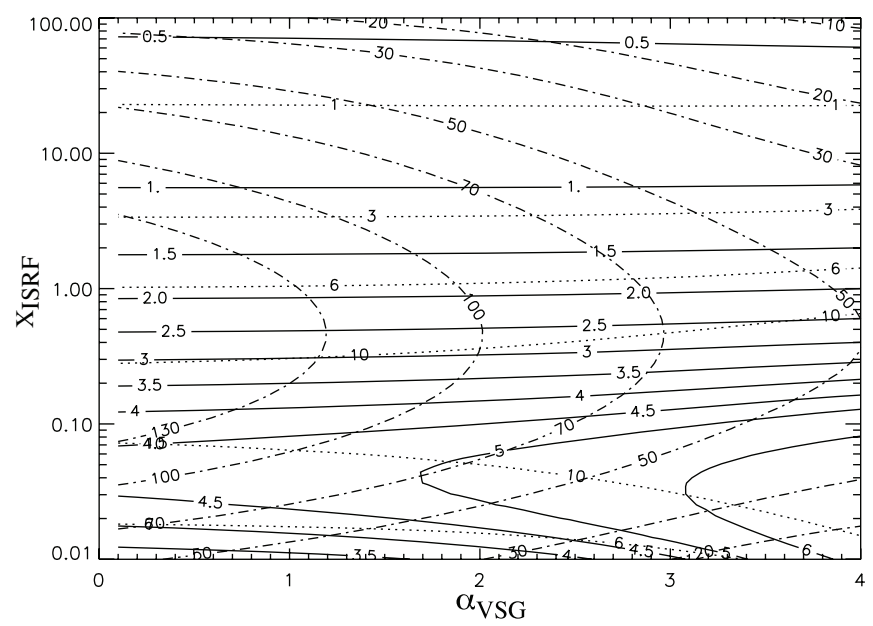

Figure 6. $I_{160} / I_{100}$ (solid lines), $I_{160} / I_{70}$ (dot lines), and $I_{160} / I_{24}$ (dash-dotted lines) deduced from the Désert et al. (1990) model as a function of the intensity of the ISRF ( $\left.X_{\mathrm{ISRF}}\right)$ and of the index of the VSG size distribution $\left(\alpha_{\mathrm{VSG}}\right)$.

where $Y_{\mathrm{BG}}, Y_{\mathrm{VSG}}$ and $Y_{\mathrm{PAH}}$ correspond to the mass abundances for each dust component. The $I_{v}$ values for each dust component in Equation (3) are computed using the version of the Désert et al. (1990) model described in Section 4.1. The intensities are computed in the photometric channel of the instruments considered in the study and have been corrected for the color correction factor, computed using the filter transmission and flux convention of each instrument used. The mass abundances are determined by fitting to the data.

Bernard et al. (2008) have shown that a significant emission excess is present at $70 \mu \mathrm{m}$ in the LMC (see their Section 5.3). This excess has also been found in the SMC by Bot et al. (2004). In the LMC, it is apparently associated with the neutral phase of the ISM (Bernard et al. 2008) and can be attributed to regions of enhanced VSG abundance. Bernard et al. (2008) studied different possible origins to explain the $70 \mu \mathrm{m}$ excess. The most likely explanation is variations of the VSG size distribution, and the excess indicates a greater abundance of large VSGs than in our Galaxy. In the context of the model we use, the VSG size distribution is given by

$$
\frac{d n}{d a} \propto a^{-\alpha_{\mathrm{VSG}}},
$$

where $d n$ is the numerical density of grains with a dimension between $a$ and $a+d a$, and $\alpha_{\mathrm{VSG}}$ is the VSG size distribution. In the Galaxy, Désert et al. (1990) derived an average value of 2.6. Bernard et al. (2008) found that a value of $\alpha_{\mathrm{VSG}} \approx 1$ is required to explain the LMC observations.

Using the Désert et al. (1990) model, we can study the variations of the different brightness ratios as a function of the $X_{\text {ISRF }}$ and $\alpha_{\mathrm{VSG}}$. Figure 6 shows the evolution of the three ratios $I_{160} / I_{100}, I_{160} / I_{70}$, and $I_{160} / I_{24}$ for different values of the $X_{\text {ISRF }}$ and $\alpha_{\mathrm{VSG}}$ derived using the model. The figure shows that $\alpha_{\mathrm{VSG}}$ does not significantly affect the $I_{160} / I_{100}$ and $I_{160} / I_{70}$ ratios for $X_{\text {ISRF }}$ values larger than about 0.1 . For our study, we do not have enough spectral coverage to warrant extra free parameters, so we decided to consider only two values of $\alpha_{\mathrm{VSG}}$ : the Galactic value $\left(\alpha_{\mathrm{VSG}}=2.6\right)$ and the value used in Bernard et al. (2008; $\left.\alpha_{\mathrm{VSG}}=1\right)$.

As a first step, we computed the normalized brightnesses between $160 \mu \mathrm{m}$ and $8 \mu \mathrm{m}$ (below this wavelength the presence 

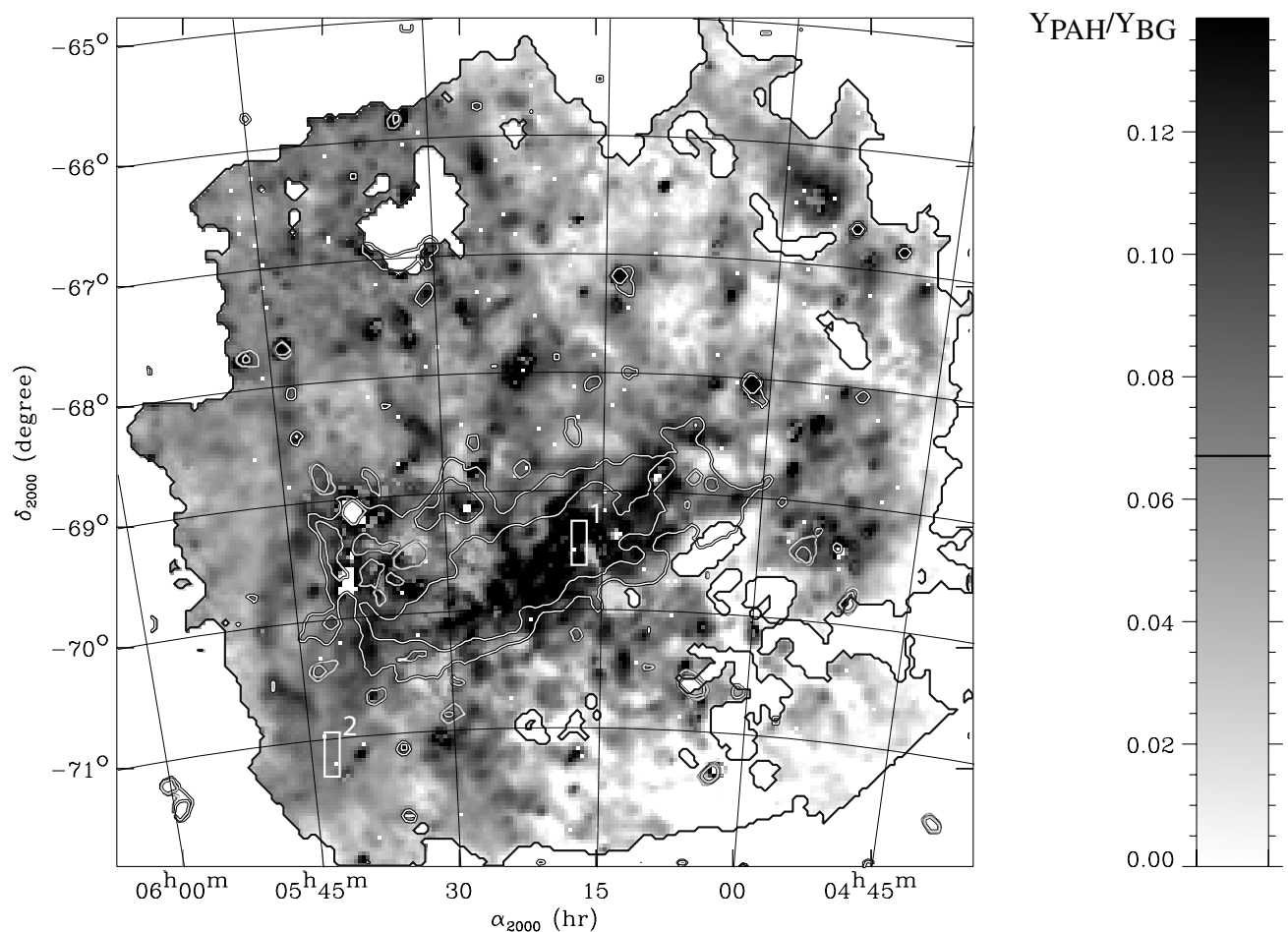

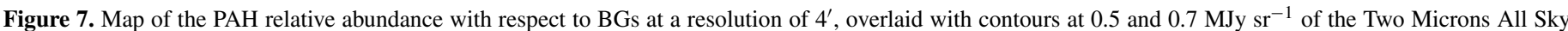

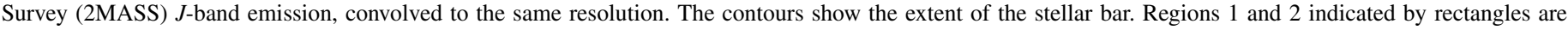
discussed in Section 5.2.1. The horizontal line in the color bar shows the value of the standard MW relative abundance.

of an additional NIR continuum is generally needed to explain the observations) for a grid of $X_{\text {ISRF }}$ values and the two selected values of $\alpha_{\mathrm{VSG}}$, using the MW dust abundances from Désert et al. (1990) (see Section 4.1). The brightness for each dust component $i$ can be written as $I_{v}^{i}=I_{v}^{i}\left(\alpha_{\mathrm{VSG}}, X_{\mathrm{ISRF}}\right)$. We obtain a table of brightnesses for the PAHs, VSGs, and BGs, separately. We compare the brightness of our infrared maps with the values in the table at each pixel of the map, in order to derive maps of the mass abundances. To ensure that the $X_{\mathrm{ISRF}}$ value is calculated correctly, we forced the model to reproduce the data at 100 and $160 \mu \mathrm{m}$ by increasing the weight of these data points in the SEDs. We derived the mass abundances minimizing the sum of the squared difference between the model and observations $\left(\chi^{2}\right)$ for each value of $X_{\mathrm{ISRF}}$ and $\alpha_{\mathrm{VSG}}$ and selected the solution with minimum $\chi^{2}$.

One shortcoming of our modeling is the assumption of a single $\mathrm{X}_{\mathrm{ISRF}}$ and therefore $\mathrm{BG}$ equilibrium temperature, along any line of sight. This is expected to be a good approximation in the LMC due to its near face-on viewing geometry. To account for the possibility of a mixture of the radiation field intensities along the line of sight, we follow the description proposed by Dale et al. (2001) assuming a power-law distribution of the dust mass $M_{d}\left(X_{\text {ISRF }}\right)$ subjected to a given ISRF intensity on a given line of sight

$$
d M_{d}\left(X_{\mathrm{ISRF}}\right) \propto X_{\mathrm{ISRF}}^{-\alpha} d X_{\mathrm{ISRF}} \quad 0.3<X_{\mathrm{ISRF}}<10^{5},
$$

where $\alpha$ is a parameter that represents the relative contribution of radiation field intensities, in the range $\alpha=1-2.5$. We first compute emission spectra using the Désert et al. (1990) model at various $X_{\mathrm{ISRF}}$ values (noted $I_{v}^{\bmod }\left(X_{\mathrm{ISRF}}\right)$ ), then sum them over
$X_{\text {ISRF }}$ according to

$$
I_{v}^{\mathrm{tot}}=\frac{\sum_{X_{\mathrm{ISRF}}} I_{v}^{\mathrm{mod}}\left(X_{\mathrm{ISRF}}\right) \times X_{\mathrm{ISRF}}^{-\alpha}}{\sum_{X_{\mathrm{ISRF}}} X_{\mathrm{ISRF}}^{-\alpha}}
$$

We follow the same methodology as described previously, constructing a table of normalized brightnesses for different $\alpha$ values for each grain component, using the MW abundance values from Désert et al. (1990) as a reference. We use the same values of $\alpha_{\mathrm{VSG}}$ as derived previously and we minimize $\chi^{2}$ in each pixel with respect to the above table, determining $\alpha$. The abundances of the various dust components are then obtained in the same way as previously.

\subsection{Results}

The objective of our modeling is to determine the distribution of the VSG and PAH abundance compared to the BG abundance across the LMC. In order to avoid any confusion between the dust emission and unresolved stars in the $8 \mu \mathrm{m}$ map which could have been left after the initial $8 \mu \mathrm{m}$ point-source subtraction, we also masked the point sources from the initial $3.6 \mu \mathrm{m}$ map, and smoothed the map to $4^{\prime}$. The median ratio between the pointsource fluxes at $3.6 \mu \mathrm{m}$ and at $8 \mu \mathrm{m}$ is 4 . In order to remove potential contamination by unresolved stars at $8 \mu \mathrm{m}$, we scaled the $3.6 \mu \mathrm{m}$ map by a factor $1 / 4$ and subtracted it from the $8 \mu \mathrm{m}$ map, assuming negligible ISM emission at $3.6 \mu \mathrm{m}$. This is an important step, specially in the stellar bar where the star density is high and where we identify a higher PAH abundance derived from the $8 \mu \mathrm{m}$ emission map (see Section 5.2.1).

Figures 7 and 8 show the spatial distribution of the $\mathrm{PAH}$ and VSG abundances relative to BGs. It can be seen that the distributions of these two dust components are quite different. 

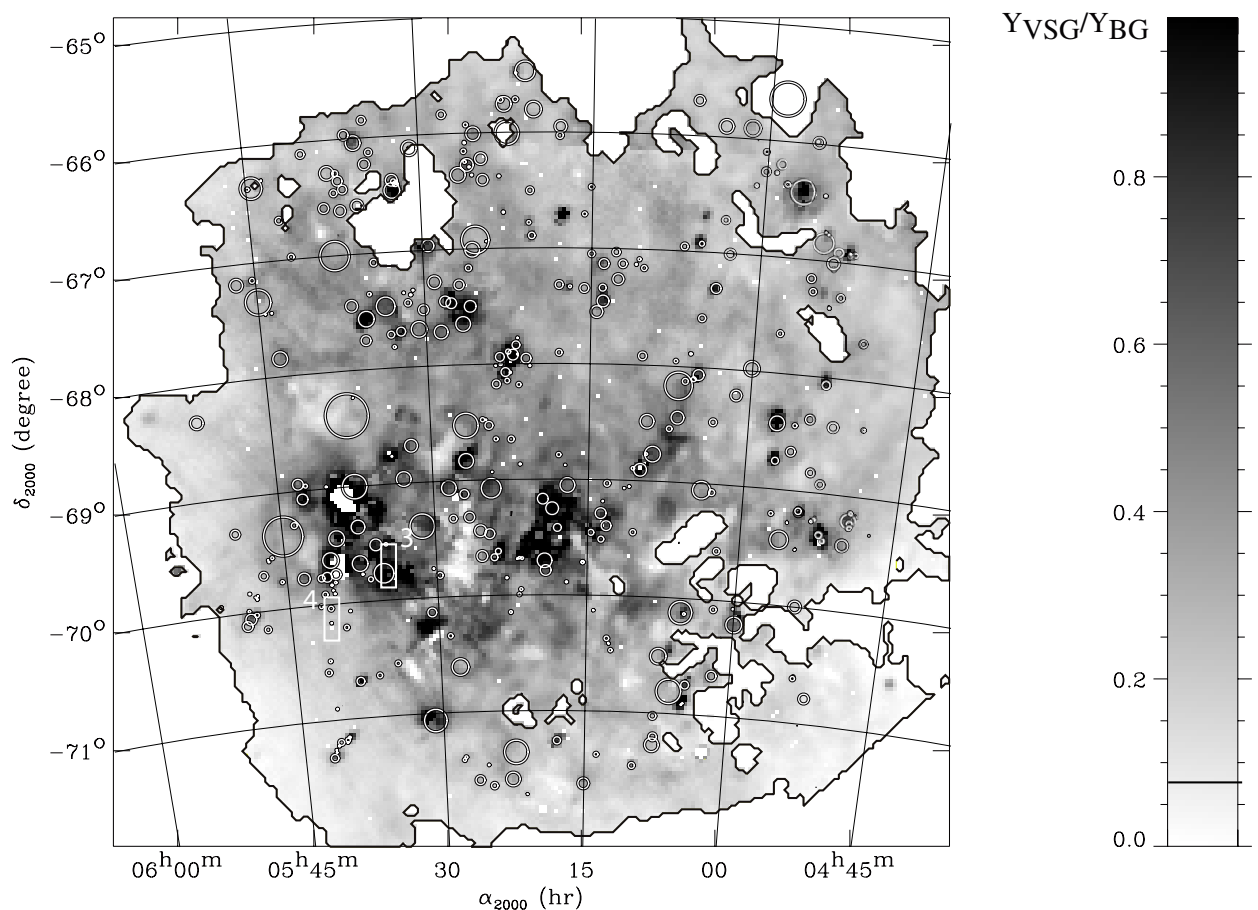

Figure 8. Map of the VSG relative abundance with respect to BGs. The overlaid symbols show the H in regions (Davies et al. 1976, catalog). Regions 3 and 4 indicated by rectangles are described in Table 2 . The horizontal line in the color bar shows the value of the standard MW relative abundance.
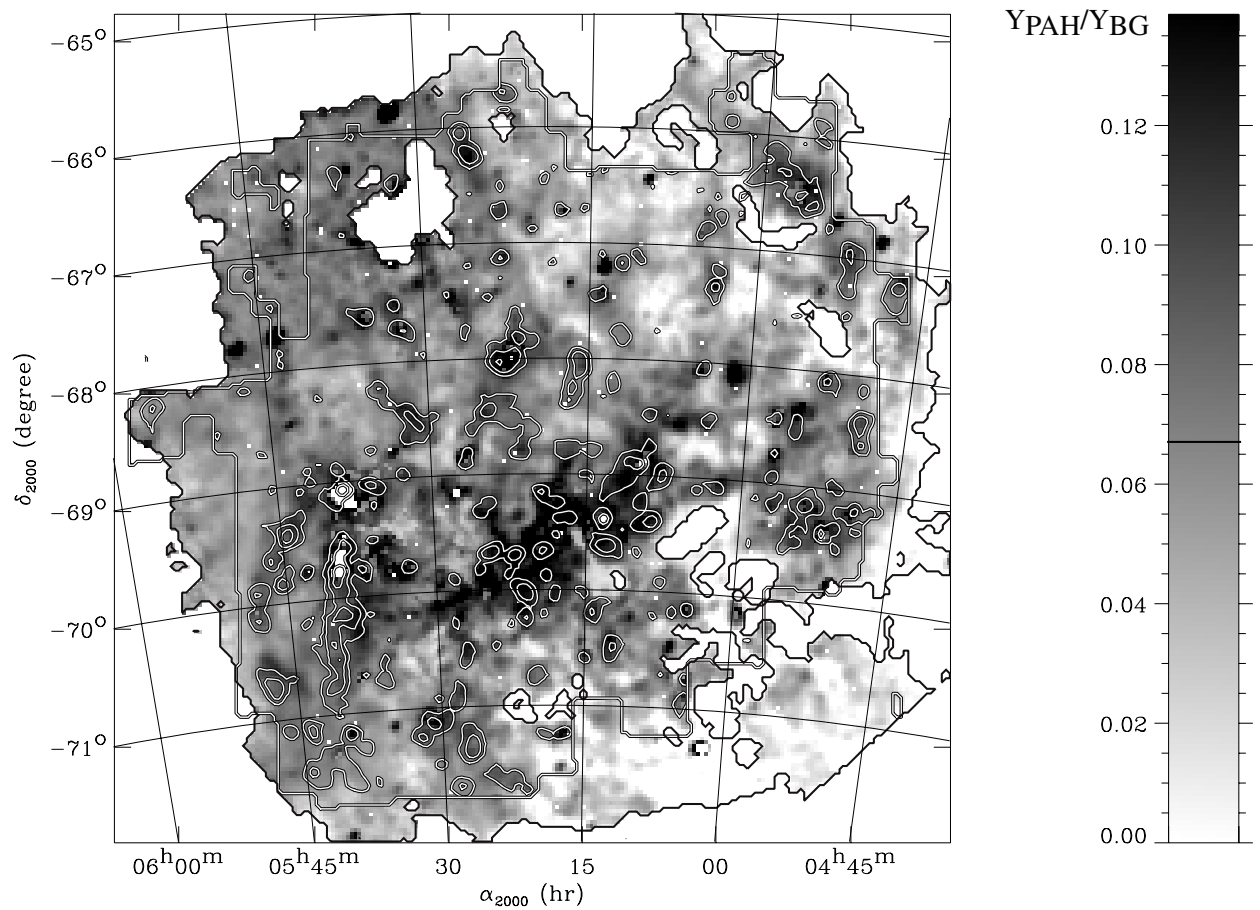

Figure 9. Map of the PAH relative abundance with respect to BGs, overlaid with the NANTEN ${ }^{12} \mathrm{CO}(J=1-0)$ integrated intensity contours convolved to the $4^{\prime}$ resolution, at $0.4,2$, and $6 \mathrm{~K} \mathrm{~km} \mathrm{~s}^{-1}$. The lines indicate the limits of the survey. The horizontal line in the color bar shows the value of the standard MW relative abundance.

The white regions around 30-Doradus have very high $\chi^{2}$ values and have been masked in all the maps. In the following sections, we analyze separately the variations of the relative abundances of each dust component.

\subsubsection{PAH Distribution}

Figure 7 shows that the PAH relative abundance is larger than average over a large elongated region toward the center of the
LMC. This region is located within the old-population stellar bar, delineated in the figure by the 2MASS contours. The PAH relative abundance increases by a factor of $\sim 2$ inside this region, compared to the rest of the LMC. Figure 9 shows that the PAH distribution is also sometimes well correlated with the molecular clouds. This result is in agreement with Bernard et al. (2008) who proposed that this may be due to the presence of enriched PAH halos similar to those found in the solar neighborhood, mostly unresolved at the distance of the LMC. Our results 
also show increased PAH abundance toward some $\mathrm{H}$ II regions. This could result from the fact that $\mathrm{H}$ II regions are generally associated with molecular clouds. We also note that $\mathrm{H}$ II regions with high $X_{\text {ISRF }}$ show low values of the PAH abundance, in agreement with findings in the MW that PAHs are deficient in warm $\mathrm{H}$ II regions. Note also the presence in Figure 7 of a northsouth elongated region between 30-Doradus and the bar (around $\alpha=5^{\mathrm{h}} 27^{\mathrm{m}}$ ) with lower apparent PAH abundance. This is likely an artifact of the method used. This region was shown in Bernard et al. (2008) to have an increased BG temperature, which they attributed to an artifact in the IRAS data at $100 \mu \mathrm{m}$ following crossing of the bright 30-Doradus region along the IRAS scans. This results in an overestimated ISRF and an underestimated PAH abundance. A similar effect is seen in Figure 8 for the VSG abundance. We also investigated whether the increase of the PAH relative abundance in the stellar bar could be due to a difference in the ISRF spectrum compared to what we used in the model. Indeed, the ISRF in the bar is likely to be dominated by the many old-population stars and should be redder than in the rest of the LMC. Since BGs are heated equally by UV and VIS photons, our procedure to derive the radiation field intensity from their temperature would lead to overestimating the UV part of the ISRF in that case. Since PAHs are expected to be mostly sensitive to UV photons, this in turn would lead to underestimating their abundance. We, however, observe an opposite behavior. We therefore can exclude that the increased PAH abundance in the stellar bar could be due to this effect.

The top panel of Figure 10 shows the median SED, computed in regions 1 and 2 (see Figure 7) with low and high PAH relative abundance $\left(Y_{\mathrm{PAH}} / Y_{\mathrm{BG}}=0.076\right.$ and 0.16$)$, respectively. The SEDs are compared to the prediction of the model described by Equation (3). The error bars correspond to the standard deviation in the background region (same background region as described in Section 3). We took into account the point-source subtraction in the error estimate by dividing the standard deviations by a mask whose values ranged between 0 and 1 for pixels with or without contamination by point sources, respectively. Table 2 summarizes the coordinates, size, brightness values, and model predictions for each region. We can see that the $160 \mu \mathrm{m}$ brightness for the two regions are within $15 \%$ of each other, while the difference in brightness at $8 \mu \mathrm{m}$ is a factor of 2, which illustrates why the model fits give $Y_{\mathrm{PAH}} / Y_{\mathrm{BG}}$ higher by a factor of 2 for region 1 . However, we can see that the model for the two SEDs is not able to reproduce the IRIS data at $12 \mu \mathrm{m}$. The model we consider here assumes a fixed fraction of neutral and ionized PAHs of $90 \%$ and $10 \%$, respectively. Therefore, we suspect that the difference between the data and model at $12 \mu \mathrm{m}$ could be due to this limitation of the model. We will discuss this point in Section 6.

\subsubsection{VSG Distribution}

Figure 8 shows the VSG relative abundance map. The VSG distribution across the LMC is different from that of the PAHs. Regions with enhanced VSGs are located around 30-Doradus and in a patch near the center of the LMC. As for he PAHs, the increase of the VSG abundance near the center of the bar cannot come from a reddened ISRF, since this would produce a decrease instead of an increase of their abundance. As opposed to the PAH abundance, the VSG relative abundance follows the distribution of the $\mathrm{H}$ II regions quite well (symbols in Figure 8). Note that a similar artifact as in the PAH abundance is evidenced in the north-south region between 30-Doradus and the bar (see Section 5.2.1 for details).

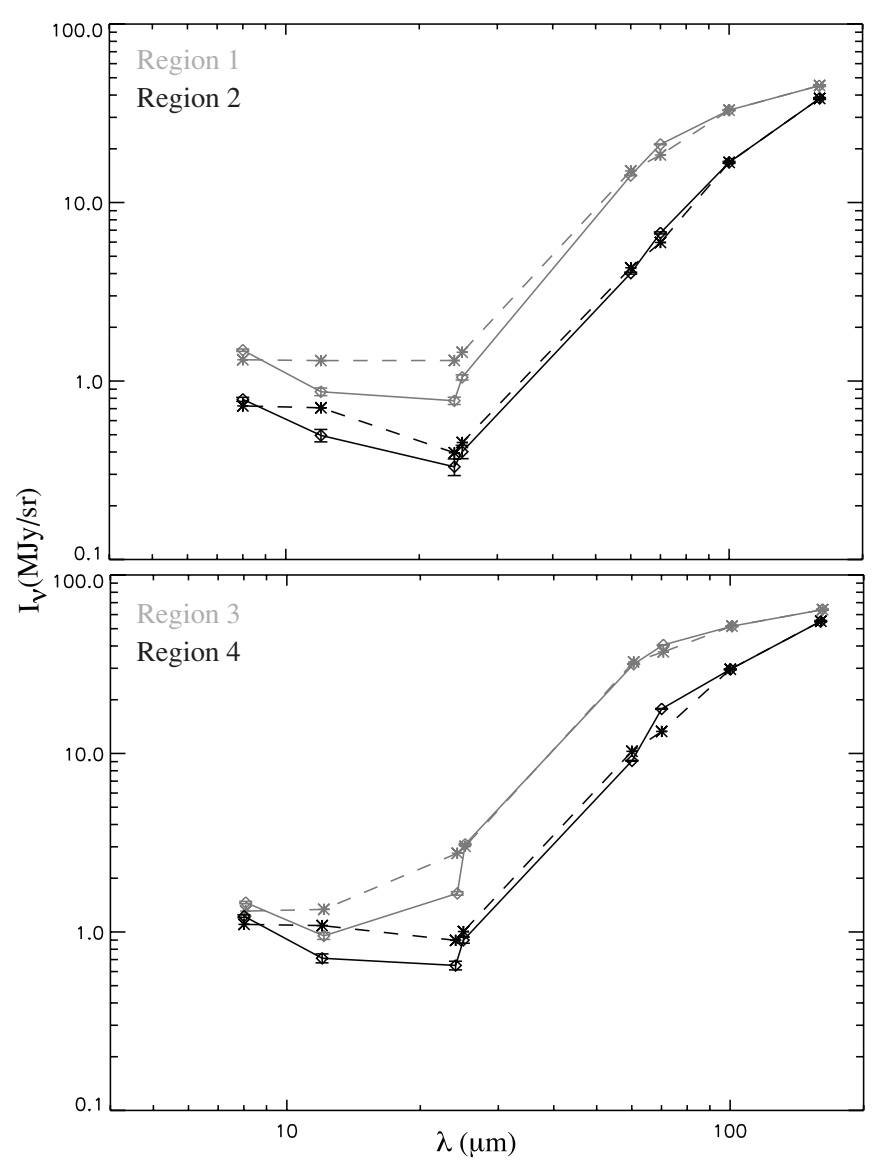

Figure 10. Median brightnesses between 5.8 and $160 \mu \mathrm{m}$ deduced from the data (diamond linked by continuous line) and brightnesses deduced from the model (stars linked by dashed line). The top panel corresponds to SEDs in regions 1 and 2 (see Figure 7) with a low and high PAH relative abundance in black and gray, respectively. The bottom panel corresponds to values in regions 3 and 4 (see Figure 8) with low and high VSG relative abundance in black and gray, respectively.

The bottom panel of Figure 10 shows the median spectra for two regions (regions 3 and 4) with low and high VSG relative abundance $\left(Y_{\mathrm{VSG}} / Y_{\mathrm{BG}}=0.24\right.$ and 1.18), respectively and their corresponding models. The main difference between the two spectra in the FIR domain (from 60 to $160 \mu \mathrm{m}$ ) is the flatness of the spectrum in region 3 with high VSG relative abundance. The $\alpha_{\mathrm{VSG}}$ value is equal to 1 for the model spectra, but the derived VSG relative abundance is increased by a factor of 2 from region 4 to 3. As discussed in Section 5.1, we only allow two values for the VSG size distribution slope, $\alpha_{\mathrm{VSG}}=1$ or the Galactic value, 2.6. For most pixels, the value deduced from the fit is 1, but some regions require the Galactic value. This is the case of high $X_{\text {ISRF }}$ regions such as 30-Doradus and some individual $\mathrm{H}$ II regions. In these warm regions, the $70 \mu \mathrm{m}$ emission is actually dominated by warm BG emission and little additional emission from VSGs is required. In those cases, the model with $\alpha_{\mathrm{VSG}}=2.6$ sometimes overestimates the $70 \mu \mathrm{m}$ emission, which could indicate the presence of a steeper VSG size distribution than in the MW. In summary, results of the fits give $\alpha_{\mathrm{VSG}}=1$ in the overall LMC except for $\mathrm{H}$ II regions where the Galactic value (or higher value) is needed.

\subsubsection{ISRF Mixture Results}

The $\alpha$ parameter (radiation field probability distribution) used in Equation (5) ranges from 1 to 2.5. Dale et al. (2001) describe 
Table 2

SEDs and Results of the SED Fits for the Four Selected Regions: 1, High $\frac{Y_{\mathrm{PAH}}}{Y_{\mathrm{BG}}} ; 2$, Low $\frac{Y_{\mathrm{PAH}}}{\mathrm{Y}_{\mathrm{BG}}} ; 3$, High $\frac{Y_{\mathrm{VSG}}}{Y_{\mathrm{BG}}} ; 4$, Low $\frac{Y_{\mathrm{VSG}}}{Y_{\mathrm{BG}}}$

\begin{tabular}{|c|c|c|c|c|}
\hline Region & 1 & 2 & 3 & 4 \\
\hline$\alpha_{2000}$ & $05: 23: 00$ & $05: 43: 00$ & 05:35:00 & 05:42:00 \\
\hline$\delta_{2000}$ & $-69: 30: 00$ & $-71: 10: 00$ & $-69: 47: 00$ & $-70: 15: 00$ \\
\hline Box size $\left(^{\circ}\right)$ & $0.4 \times 0.4$ & $0.4 \times 0.4$ & $0.4 \times 0.4$ & $0.4 \times 0.4$ \\
\hline$I_{160}\left(\mathrm{MJy} \mathrm{sr}^{-1}\right)$ & $46.72 \pm 0.49$ & $40.30 \pm 0.47$ & $66.05 \pm 0.51$ & $56.78 \pm 0.48$ \\
\hline$I_{100}\left(\mathrm{MJy} \mathrm{sr}^{-1}\right)$ & $34.06 \pm 0.23$ & $17.68 \pm 0.22$ & $53.34 \pm 0.24$ & $30.51 \pm 0.22$ \\
\hline$I_{70}\left(\mathrm{MJy} \mathrm{sr}^{-1}\right)$ & $21.91 \pm 0.10$ & $7.12 \pm 0.09$ & $41.90 \pm 0.10$ & $18.32 \pm 0.09$ \\
\hline$I_{60}\left(\mathrm{MJy} \mathrm{sr}^{-1}\right)$ & $14.67 \pm 0.05$ & $4.23 \pm 0.05$ & $32.70 \pm 0.05$ & $9.38 \pm 0.05$ \\
\hline$I_{25}\left(\mathrm{MJy} \mathrm{sr}^{-1}\right)$ & $1.08 \pm 0.04$ & $(4.23 \pm 0.36) 10^{-1}$ & $3.20 \pm 0.04$ & $(9.28 \pm 0.36) 10^{-1}$ \\
\hline$I_{24}\left(\mathrm{MJy} \mathrm{sr}^{-1}\right)$ & $(8.02 \pm 0.37) 10^{-1}$ & $(3.48 \pm 0.37) 10^{-1}$ & $1.70 \pm 0.04$ & $(6.69 \pm 0.37) 10^{-1}$ \\
\hline$I_{12}\left(\mathrm{MJy} \mathrm{sr}^{-1}\right)$ & $(9.01 \pm 0.44) 10^{-1}$ & $(5.22 \pm 0.42) 10^{-1}$ & $(9.81 \pm 0.43) 10^{-1}$ & $(7.34 \pm 0.42) 10^{-1}$ \\
\hline$I_{8}\left(\mathrm{MJy} \mathrm{sr}^{-1}\right)$ & $1.54 \pm 0.02$ & $(8.31 \pm 0.22) 10^{-1}$ & $1.51 \pm 0.02$ & $1.26 \pm 0.02$ \\
\hline$Y_{\mathrm{PAH}} / Y_{\mathrm{BG}}$ & $1.4110^{-1}$ & $7.2710^{-2}$ & $1.3710^{-1}$ & $8.3110^{-2}$ \\
\hline$Y_{\mathrm{VSG}} / Y_{\mathrm{BG}}$ & $4.8710^{-1}$ & $1.2210^{-1}$ & 1.09 & $2.2810^{-1}$ \\
\hline$X_{\mathrm{ISRF}}$ & 0.85 & 0.49 & 0.28 & 0.59 \\
\hline
\end{tabular}

the two extreme cases: $\alpha=2.5$ corresponds to diffuse regions, whereas $\alpha=1$ represents photodissociation regions near young stars. In the LMC, regions with $\alpha=2.5$ are more common. The decrease of the parameter $\alpha$ across the LMC follows the increase of the BG equilibrium temperature (see Figure 7 of Bernard et al. 2008).

The PAH and VSG relative abundances derived for the case where we let the ISRF strength mixture to vary are given in Figures 11 and 12, respectively. The scale in the two maps are the same as in Figures 7 and 8. Figure 11 still shows the increase of the PAH abundance in the old-population stellar bar, although the amplitude of the variation is slightly less than that in Figure 7. The VSG relative abundance map is quite similar to the one shown in Figure 8: the VSG relative abundance still follows roughly the distribution of the $\mathrm{H}$ II regions and the main region of enhanced VSG abundance is still located around 30-Doradus and near the center of the LMC. Results do not appear to change significantly when we consider the effect of a realistic ISRF strength mixture along the line of sight.

\section{DISCUSSION}

PAHs and VSGs could have a different origin or could be subjected to different processing in the ISM. Several studies of the MIR aromatic bands and continuum emission around $15 \mu \mathrm{m}$ showed that PAH and VSG emission are linked to star formation activity. Forster Schreiber et al. (2004) describe the PAH component as representative of the quiescent environments while the VSG component traces the active star formation sites. They attribute the disappearance of PAH bands in $\mathrm{H}$ II regions to the destruction of PAHs in regions with high intensity of the radiation field (in agreement with the decrease of the IRAS 12/ 100 ratio in regions of intense UV radiation, from Boulanger et al. 1988). Povich et al. (2007) considered two possible mechanisms for PAH destruction in H II regions: stellar UV photons and $\mathrm{X}$ rays. They concluded that only UV photons could be responsible for the absence of PAHs in these regions. Contursi et al. (2000) examined the N4 region in the LMC using ISOCAM data and found that the maximum emission is located at the interface between the $\mathrm{H}$ II region and the molecular cloud, as was also found from observations of M17 in our Galaxy by Cesarsky et al. (1996). Helou et al. (2004) studied the spiral galaxy NGC 300 using Spitzer data and found that emission at $24 \mu \mathrm{m}$ traces the star-forming regions whereas the emission at $8 \mu \mathrm{m}$ highlights the edges of these regions. However, interpreting those observations in terms of VSG abundance remains difficult, since the excitation conditions are generally poorly constrained.

At least a fraction of the PAH population is thought to be formed and injected into the ISM by the stellar winds following mass loss from old carbon-rich stars. Loup et al. (1999) conducted a survey of $0.5 \mathrm{deg}^{2}$ in the bar of the LMC using the ISOCAM and DENIS instruments. Their objective was to build a complete sample of thermally pulsing asymptotic giant branch (TP-AGB) stars. During this phase, stars lose mass and carbon-rich AGB could contribute significantly to the formation of PAHs. They found approximately 300 TP-AGB stars, of which $9 \%$ have high mass-loss rates. Some studies of postAGB stars in transition between the AGB and planetary nebulae phase exhibit signatures of dust attributed to PAHs (e.g., Venkata Raman \& Anandarao 2008). Justtanont et al. (1996) analyzed MIR spectroscopy in three post-AGB stars, known to exhibit an emission feature at $21 \mu \mathrm{m}$. In addition to a weak emission at $11.3 \mu \mathrm{m}$, they found several features which match the emission bands in the spectrum of chrysene $\left(\mathrm{C}_{8} \mathrm{H}_{12}\right)$, which is one of the simplest PAH molecules. The $21 \mu \mathrm{m}$ emission feature has never been observed in carbon-rich AGB stars, nor planetary nebulae. The PAH molecule responsible for this feature could be formed in the dust shells around some post-AGB stars and lead to increase the abundance of this PAH species (Justtanont et al. 1996).

In this study, we showed that the PAH relative abundance in the LMC is maximum in an extended region corresponding to the old-population stellar bar, a region with no strong current stellar formation activity. To our knowledge, this is the first time such an effect is evidenced at Galactic scale. We interpret this finding as an indication that PAHs may have formed efficiently in this region in the past from carbon-rich stars winds and have remained overabundant in the surrounding ISM. Our result also shows the presence of a region with a significantly increased VSG abundance in the same region, although with a lesser spatial extent. This is likely to reveal that VSGs also formed efficiently from the same process. The difference in the spatial extent of the regions could either reflect specific formation processes or differences between the evolution of the two species in the ISM. In the later hypothesis, our result would suggest that PAHs are in fact more resistant than VSGs to destruction processes in the diffuse ISM.

A careful examination of Figure 13 which compares the spatial distribution of PAHs and VSGs indicates that there are other regions where the $\mathrm{PAH}$ and VSG abundances are 

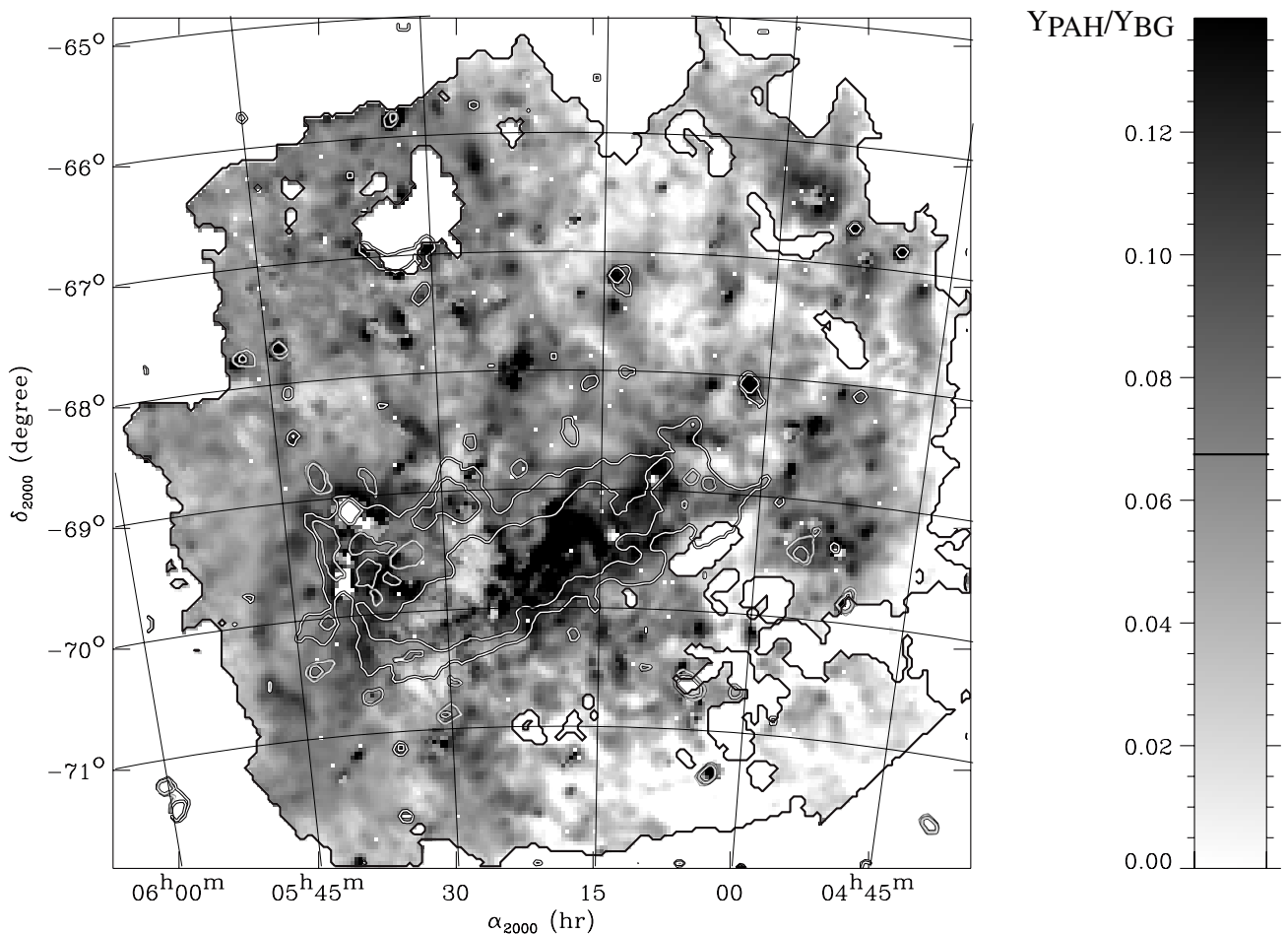

Figure 11. Map of the PAH relative abundance with respect to BGs, for the case of an ISRF mixture along the line of sight. Contours correspond to the Two Microns All Sky Survey (2MASS) J-band map, convolved to the $4^{\prime}$ resolution. The horizontal line in the color bar shows the value of the standard MW relative abundance.
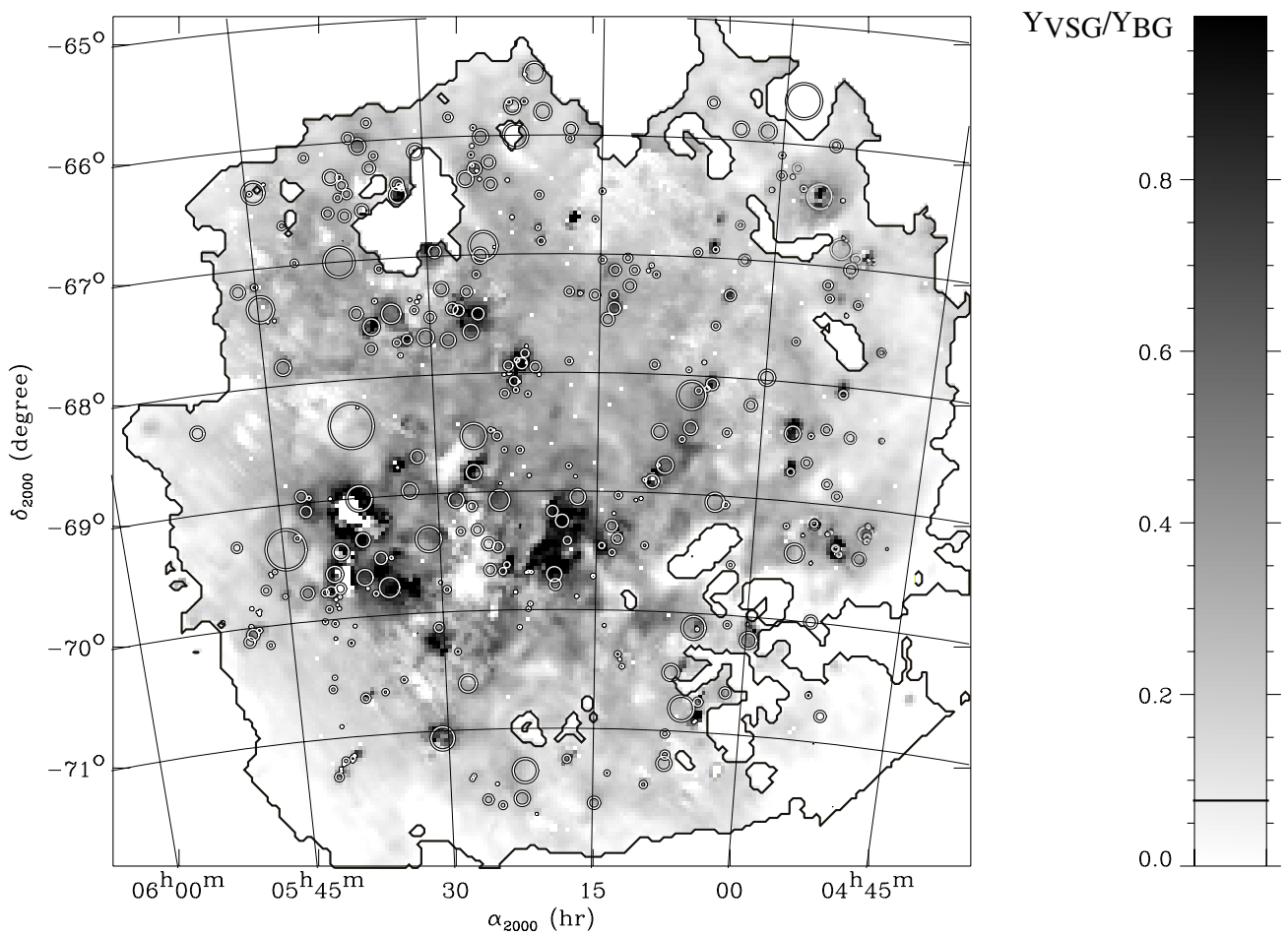

Figure 12. Map of the VSG relative abundance with respect to BGs, for the case of an ISRF mixture along the line of sight. The overlaid symbols show the H II regions (Davies et al. 1976, catalog). The horizontal line in the color bar shows the value of the standard MW relative abundance.

spatially correlated. Most noticeably, the region surrounding 30-Doradus and its extension to the southwest both have enhanced PAH and VSG abundances. However, significant differences are also apparent. For instance, the region of enhanced PAH abundance to the west of the main molecular ridge, south of 30-Doradus, as well as the arc-like molecular ridge to the east have no counterpart in the VSG abundance map. Similarly, at the northwest end of the stellar bar, the PAH and VSG abundance maps appear anticorrelated.

In the solar neighborhood, such independent variations of the distribution of PAHs and VSGs have been observed. For instance, Bernard et al. (1993) showed the existence of increased 

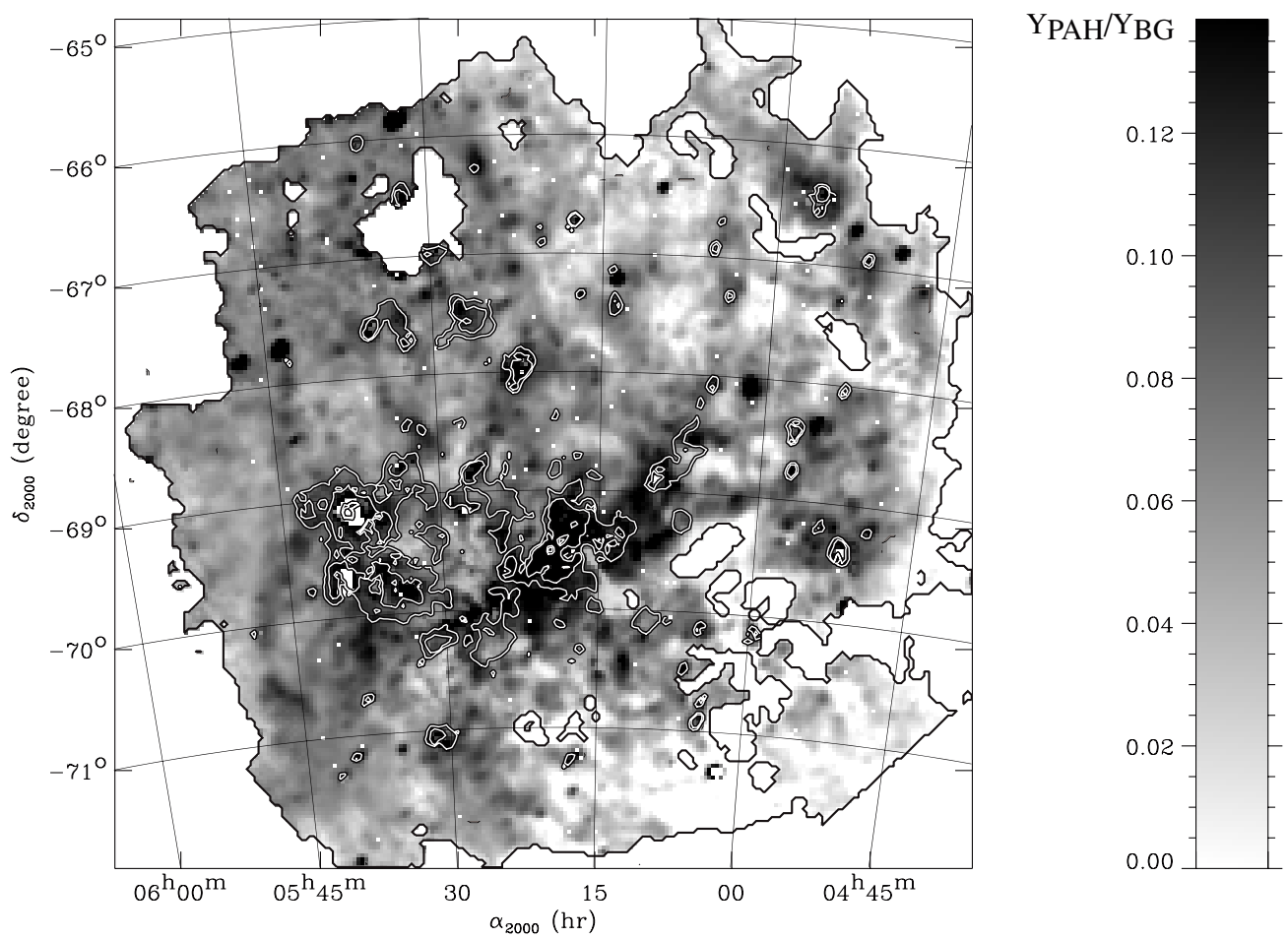

Figure 13. Map of the PAH relative abundance with respect to BGs, overlaid with contours at 0.6 and 0.9 of the VSG relative abundance.

PAH abundance regions at the periphery of some translucent clouds. This could result from the destruction of larger species such as VSGs into PAH molecules, induced by the action of external low-velocity shocks at the surface of the clouds. Miville-Deschênes et al. (2002) evidenced large variations of the PAH emission in the Ursa Major cirrus. They proposed that the observed color variations are due to actual PAH abundance variations due to grain shattering in energetic graingrain collisions induced by turbulence or grain coagulation caused by small turbulent motions. Therefore, regions with large turbulent motion could generate PAHs through shattering of larger dust particles, such as VSGs. Indeed, the destruction of graphitic dust is expected to lead to the formation of large two-dimensional PAH-like molecules. Berné et al. (2007) studied a photodissociation region using the Spitzer IRS data and found a disappearance of VSG emission while the PAH emission increases. Like Cesarsky et al. (2000) they suggested the possibility that VSGs could evolve into PAHs under UV photon driven processes. Under this hypothesis, VSGs can be viewed as PAH clusters. Although at the distance of the LMC, such small-scale structure would not be resolved, it is likely that the differences we evidence between the spatial distribution of PAHs and VSGs are of a similar nature. It is worth noticing that, in areas with no VSG enhancement such as the southern tip of the arc-shaped molecular ridge, the PAH abundance enhancement appears to trace the surface of the molecular clouds, a similar situation as that observed in nearby halo clouds, although with much larger physical scale, given the distance of the LMC.

Similar variations were observed in the SMC by Bolatto et al. (2007) who found large variations of the $I_{8} / I_{24}$ ratio across the southwest region of the bar. They attributed these to spatial variations of the PAH abundance, with the PAH abundance decreasing with increasing $24 \mu \mathrm{m}$ brightness. They suggested that the PAH abundance variations are essentially driven by the local ISRF and PAH photodestruction. In the
LMC, Bernard et al. (2008) showed that a large region around of 30-Doradus has excess $70 \mu \mathrm{m}$ emission, which they interpreted through the flattening of the VSG size distribution, potentially caused by the erosion of larger BGs by shocks propagating from the 30-Doradus region. In our study, we find that this same region also has an increased VSG abundance. Our result shows that this region, in addition to showing an increase of the largest VSGs also shows an increase of the overall VSG abundance. Bernard et al. (2008) indicated that the increase of the largest VSG abundance would correspond to $\simeq 13 \%$ of the BG mass, which converts into a VSG abundance increase of $77 \%$ for a standard MW dust mixture. The excess we measure in this region corresponds to an increased abundance by a factor of about 2.4, and is therefore larger than that imputable to VSG size distribution change only. Similarly, Lisenfeld et al. (2002) analyzed the emission from NGC 1569, a dwarf galaxy with a metallicity between those of the LMC and the SMC. Using the Désert et al. (1990) model, they evidenced an enhancement of the VSG abundance relative to BGs, with respect to the solar neighborhood, by a factor of $2-7$, depending on the model parameters they used. They explain this result by the BG destruction due to shocks in the turbulent ISM of this galaxy.

We therefore consider it likely that the differences evidenced here between the spatial distribution of the PAH and VSG abundances relative to BGs are tracing both the production of PAHs from the destruction of VSGs at the surface of molecular clouds, and the production of VSGs from the destruction of larger grains in shocks.

We saw in Figure 10 that the model with neutral PAHs was not able to reproduce the IRIS data at $12 \mu \mathrm{m}$. We suspect that this is due to the ionization state of the PAHs or the nature of the molecules, such as dehydrogenation. It is difficult to constrain the nature of the PAHs in the LMC from the data presented here only. However, varying the PAH ionization fraction in the model, we find that the model requires $100 \%$ of the PAHs in the ionized 


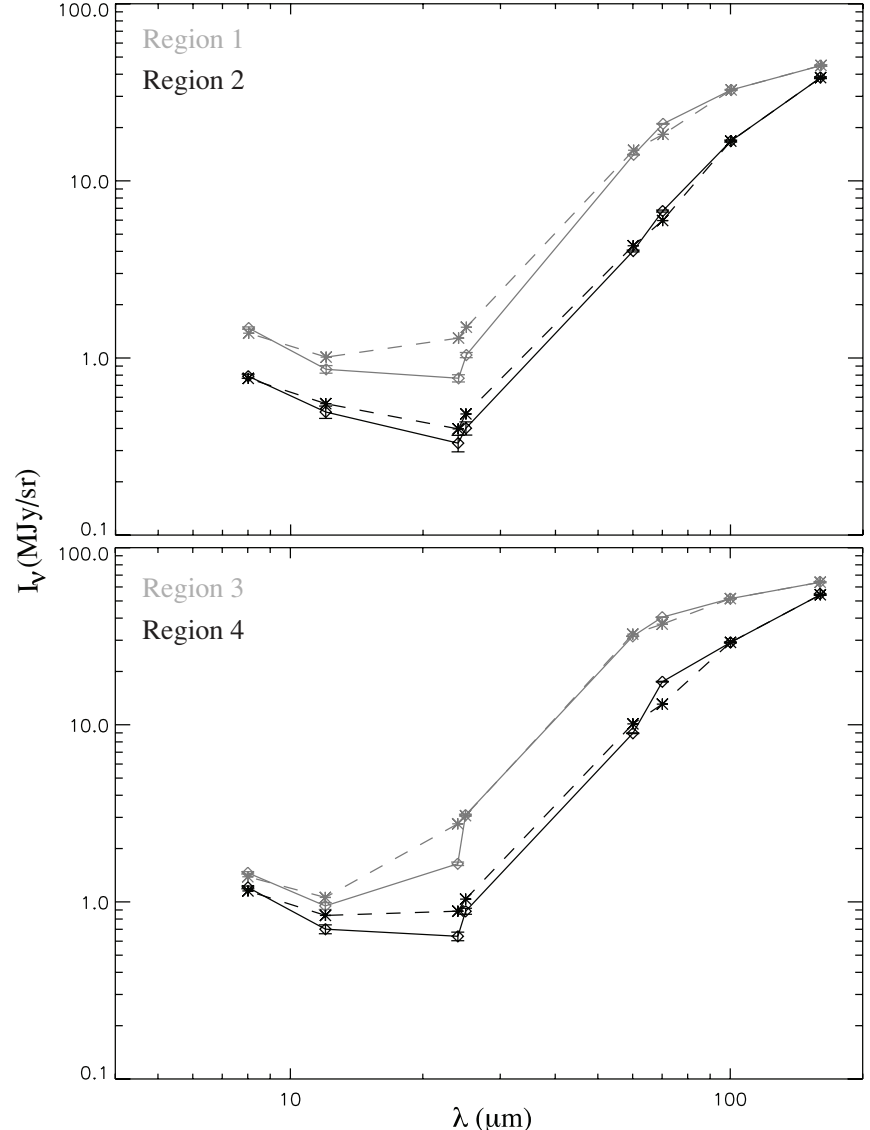

Figure 14. Same figure as Figure 10, using a model with $100 \%$ ionized PAHs.

state to explain the observations at $12 \mu \mathrm{m}$ (see Figure 14). Even if this model does not reproduce the data at $12 \mu \mathrm{m}$ perfectly, the difference between the model and the data is smaller than with our initial hypothesis. Flagey et al. (2006) obtained a neutral over ionized PAHs ratio close to 50\% in the MW (GLIMPSE field). Therefore, PAHs in the LMC could be more ionized than in the Galactic plane. However, the exact nature of PAHs in the LMC could be different than in our Galaxy. A study of the nature of the PAH molecules is essential to determine the origin of the observed difference at $12 \mu \mathrm{m}$ between our Galaxy and the LMC. Such study may become possible using the data from the Spitzer Sage-Spec legacy program.

\section{CONCLUSION}

We used the data of the SAGE Spitzer legacy survey, combined with the IRIS data to measure the variations in the colors of infrared emission from dust in the LMC. Comparisons with results from the literature showed a higher $I_{8} / I_{24}$ ratio in the LMC than in other low-metallicity environments, which could result from a relatively higher PAH abundance in the LMC with respect to its metallicity class. Moreover, the $I_{3.6} / I_{8}$ and $I_{4.5} / I_{8}$ ratios are smaller in the LMC than in the MW. This could result from different properties of the near-infrared continuum or from different properties of the PAHs. In particular, PAHs could be smaller in the LMC, compared to our Galaxy, and more ionized. The SED modeling of regions with enhanced PAH and VSG relative abundances in the LMC show a similar result: the model used requires a large fraction (potentially 100\%) of ionized PAHs in order to minimize the difference between the model and the data at $12 \mu \mathrm{m}$.
In order to understand the processes of grain formation and destruction at the scale of the LMC, we constructed for the first time maps of the PAH and VSG relative abundances with respect to BGs of the overall galaxy, taking into account the distribution of the radiation field intensity, as inferred from the equilibrium temperature of the BGs. We evidenced an increase of the PAH relative abundance over an extended region which matches the extent of the old-population stellar bar. PAHs are also overabundant at the periphery of some molecular clouds. The VSG abundance is increased over a region around 30-Doradus and in a small region near the center of the bar. This clear distinction between the PAH and VSG spatial distribution could be due to different origins or different processings in the ISM for these two dust components. In agreement with several studies, the VSG abundance distribution appears to follow the star formation activity while that of PAHs could follow more quiescent environments.

We interpret the larger PAH abundance in the bar as a sign of the past formation of these species by C-rich mass losing stars. VSGs could also be formed from the same process but the difference in the spatial distribution remains unclear: the two dust components could originate from different stellar populations with different distributions, or VSGs and PAHs could have been evolved differently in the diffuse ISM. If destruction is at work, this may indicate that VSGs are actually destroyed faster than PAHs in the diffuse ISM. We interpret the rest of the abundance variations as signs of the evolution of dust species of different sizes in the ISM. The region around 30-Doradus shows increased VSG abundance, which could result from the destruction of larger BGs in shocks. Similarly, regions close to the surface of molecular clouds with an increase PAH relative abundance could result from the destruction of VSGs into PAHs by shocks impacting the cloud's surfaces.

\section{REFERENCES}

Allamandola, L. J., Tielens, A. G. G. M., \& Barker, J. R. 1989, ApJS, 71, 733

Allende Prieto, C., Lambert, D. L., \& Asplund, M. 2001, ApJ, 556, L63

Arendt, R. G., et al. 2008, ApJ, 682, 384

Bernard, J.-P., Boulanger, F., \& Puget, J.-L. 1993, A\&A, 277, 60

Bernard, J.-P., et al. 2008, ApJ, 136, 919

Berné, O., et al. 2007, A\&A, 469, 575

Bolatto, A. D., et al. 2007, ApJ, 655, 212B

Bot, C., et al. 2004, A\&A, 423, 567

Boulanger, F., et al. 1988, ApJ, 332, 328

Cesarsky, D., et al. 1996, A\&A, 315, L309

Cesarsky, D., et al. 2000, A\&A, 354, L87

Churchwell, the GLIMPSE Team 2005, RevMaxAA, 23, 53

Clayton, G. C., \& Martin, P. G. 1985, ApJ, 288, 558

Contursi, A., et al. 2000, A\&A, 362, 310

Cox, N. J. L., et al. 2006, A\&A, 447, 991

Dale, D. A., et al. 2001, ApJ, 549, 215

Davies, R. D., Elliott, K. H., \& Meaburn, J. 1976, MNRAS, 81, 89

Désert, F.-X., Boulanger, F., \& Puget, J.-L. 1990, A\&A, 237, 215

Dufour, R. J. 1984, in IAU Symp. 108, Structure and Evolution of the Magellanic Clouds, ed. S. Van den Bergh \& K. S. de Boer (Dordrecht: Kluwer), 353

Dwek, E., \& Galliano, F. 2009, The Fourth Spitzer Science Center Conference, The Evolving ISM in the Milky Way and Nearby Galaxies, ed. K. Sheth et al., 5D

Dwek, E., et al. 1997, ApJ, 475, 565

Engelbracht, C. W., et al. 2005, ApJ, 628, L29

Fazio, G. G., et al. 2004, ApJS, 154, 10

Flagey, N., et al. 2006, A\&A, 453, 969

Forster Schreiber, N. M., et al. 2004, A\&A, 419, 501

Gordon, K. D., et al. 2003, ApJ, 594, 279

Helou, G. 1986, ApJ, 311, L33

Helou, G., et al. 2004, ApJS, 154, 253

Houck, J. R., et al. 2004, ApJS, 154, 18 
Justtanont, K., et al. 1996, A\&A, 309, 612

Lisenfeld, U., Israel, F. P., Stil, J. M., \& Sievers, A. 2002, A\&A, 382, 860

Loup, C., et al. 1999, in IAU Symp. 191, Asymptotic Giant Branch Stars, ed. T. Le Bertre et al. (San Francisco, CA: ASP), 561

Matsuura, M., et al. 2008, in IAU Symp., 251, Organic Matter in Space, ed. S. Kwok \& S. A. Sandford (Cambridge: Cambridge Univ. Press), 197

Meixner, M., et al. 2006, AJ, 132, 2268

Miville-Deschênes, M. A., Boulanger, F., Joncas, G., \& Falgarone, E. 2002, A\&A, 381, 209

Miville-Deschênes, M. A., \& Lagache, G. 2005, ApJS, 157, 302

Povich, M. S., et al. 2007, ApJ, 660, 346
Rapacioli, M., Joblin, C., \& Boissel, P. 2005, A\&A, 429, 193

Rieke, G. H., et al. 2004, ApJS, 154, 25

Sakon, I., et al. 2006, ApJ, 651, 174

Sauvage, M., \& Vigroux, L. 1991, in IAU Symp. 148, The Magellanic Clouds, ed. R. Haynes \& D. Milne (Dordrecht: Kluwer), 407

Staveley-Smith, L., et al. 2003, MNRAS, 339, 87

van der Marel, R. P., \& Cioni, M. R. 2001, AJ, 122, 1807

Venkata Raman, V., \& Anandarao, B. G. 2008, MNRAS, 385, 1076

Werner, M. W., et al. 2004, ApJS, 154, 1

Westerlund, B. E. 1997, The Magellanic Clouds (New York: Cambridge Univ. Press), 122, 1807 